\title{
A Two-Level Nonconforming Rotated Quadrilateral Finite Element Method for the Stationary Navier-Stokes Equations
}

\author{
Weijun Tian, ${ }^{1,2}$ Liquan Mei $\mathbb{D}^{1}{ }^{1}$ and Yinnian $\mathrm{He}^{1}$ \\ ${ }^{1}$ Department of Computational Science, School of Mathematics and Statistics, Xi'an Jiaotong University, No. 28, \\ Xianning West Road, Xi'an, Shaanxi 710049, China \\ ${ }^{2}$ Department of Applied Mathematics, School of Mathematics and Statistics, Xianyang Normal University, No. 43, Wenlin Road, \\ Xianyang, Shaanxi 712000, China \\ Correspondence should be addressed to Liquan Mei; lqmei@mail.xjtu.edu.cn
}

Received 17 November 2021; Revised 27 December 2021; Accepted 5 January 2022; Published 23 February 2022

Academic Editor: M.I. Herreros

Copyright (c) 2022 Weijun Tian et al. This is an open access article distributed under the Creative Commons Attribution License, which permits unrestricted use, distribution, and reproduction in any medium, provided the original work is properly cited.

\begin{abstract}
In this paper, we propose a two-level nonconforming rotated finite element (TNRFE) method for solving the Navier-Stokes equations. A new nonconforming rotated finite element (NRFE) method was proposed by Douglas added by conforming bubbles to velocity and discontinuous piecewise constant to the pressure on quadrilateral elements possessing favorable stability properties. The TNRFE method involves solving a small Navier-Stokes problem on a coarse mesh with mesh size $H$ and a large linearized Navier-Stokes problem on a fine mesh with mesh size $h$ by the NRFE method. If we choose $h=O\left(H^{2}\right)$, the TNRFE method gives the convergence rate of the same order as that of the NRFE method. Compared with the NRFE method, the TNRFE method can save a large amount of CPU time. In this paper, the stability of the approximate solutions and the error estimates are proved. Finally, the numerical experiments are given, and results indicate that the method is practicable and effective.
\end{abstract}

\section{Introduction}

When the Navier-Stokes equations are discretized by finite element methods, two problems often arise: one is that the discrete inf-sup condition is broken, and the other is that the pseudo-oscillation is caused by the dominant convection term. In order to solve the first problem, we use the new nonconforming element proposed by Jim Douglas [1] added by conforming bubbles to the velocity and discontinuous piecewise constant to the pressure on quadrilateral elements which possess favorable stability properties. Brezzi and Russo [2] found that adding and eliminating bubbles to the finite element space is equivalent to the addition of a stabilizing term of a streamline diffusion type. It is equivalent to tuning up the amount of streamline artificial viscosity and corresponds to reducing the residual inside each element.

To deal with the second problem, we adopt the Newton TNRFE method which involves solving one small Navier-Stokes problem on a coarse mesh with mesh size $H$ and a large linearized Navier-Stokes problem on a fine mesh with mesh size $h$.
It is well known that the approximate space of velocity and pressure should generally satisfy the LBB condition when solving Navier-Stokes equations by finite element methods. This matching is difficult for the conforming element. For example, if the triangle element is used, the velocity is a piecewise linear element, and the pressure is a piecewise constant, then the LBB condition is not satisfied. If the velocity is changed to piecewise quadratic, then the error estimates for velocity will lose one order. If you use the following two rectangular elements, (1) the velocity is a bilinear polynomial interpolation and the pressure is a piecewise constant; (2) the velocity is double polynomial interpolation and the pressure is of bilinear form, then the LBB condition is not satisfied.

To some extent, the nonconforming element can overcome this difficulty, and it is often used in practical computation because of its simple structure, economical calculation, and error matching. The nonconforming finite element method for the Stokes problem was first proposed by Crouzeix and Raviart [3], and they used the piecewise 
linear triangle element with three midpoints as the velocity approximation space and the piecewise constant finite element as the pressure approximation space to obtain an approximation scheme (the $\mathrm{C}-\mathrm{R}$ scheme) for the Stokes problem. In this way, not only the LBB condition is satisfied but also some optimal error estimates of velocity and pressure are obtained. Temam [4] also proposed the nonconforming linear element as the approximate space of velocity, which obtained satisfactory error estimates. Because the rectangular element has the advantages of simple mesh and less bandwidth of the stiffness matrix, Rannacher and Turek [5] analyzed the rotational bilinear nonconforming element with local base span $\left\{1, x, y, x^{2}-y^{2}\right\}$. However, if the definition of the global nonconforming space which requires continuity at the midpoint of the common inner boundary of the adjacent elements is adopted, the optimal error estimates for the real quadrilateral subdivision region will not be obtained. To solve this problem, Jim Douglas et al. [1] modified the rotated bilinear local basis $\operatorname{span}\left\{1, x, y, x^{2}-y^{2}\right\}$ to $\operatorname{span}\left\{1, x, y,\left(3 x^{2}\right.\right.$ $\left.\left.-5 x^{4}\right)-\left(3 y^{2}-5 y^{4}\right)\right\}$, which has the following properties: $\left\langle 1, w_{j}-w_{k}\right\rangle_{\Gamma_{j k}}=0$ and $\left\langle 1, w_{j}\right\rangle_{\Gamma_{j}}=0$. Han [6] proposed that a nonconforming rectangular element with five degrees of freedom for the velocity, whose node parameters are the function value at the center and the midpoint of the four sides of the element, and the shape function space span $\left\{1, x, y, 1 / 2\left(5 x^{4}-3 x^{2}\right), 1 / 2\left(5 y^{4}-3 y^{2}\right)\right\}$ for the standard element $[-1,1]^{2}$ can also obtain the best order error estimate. Although the element has the same order of convergence speed as the Douglas rotation element, it has one more degree of freedom than the latter, which increases the computational load and decreases the efficiency. In solving the Stokes problem and the Navier-Stokes problem, Cai et al. $[7,8]$ used the Douglas rotation element, added a compatible bubble function to the velocity approximation space as the internal degree of freedom, and used the piecewise constant finite element space as the pressure approximation space. Cai et al. [7] gave the existence and uniqueness of the solution and the convergence rate of the nonconforming Galerkin (NFG) method when the Douglas rotation element was used for the velocity, but the numerical experiments were not given.

A part of the work of this paper is to solve the steady Stokes equations and Navier-Stokes equations by using the NRFE method, explain the existence and uniqueness of the solution and the convergence speed, and give the concrete design process and numerical experiment of the algorithm.

In practice, solving the Navier-Stokes equations is very time-consuming, so people try to save computing time as much as possible. One of them is the two-layer grid method, which is to solve a nonlinear equation on a coarse grid, and the purpose of solving a linear equation on a fine mesh is to obtain the approximate solution of the nonlinear equation in less time and to maintain the optimal convergence speed, specifically. The two-layer mesh method is based on the finite element space $X_{H}, X_{h}$ for the velocity approximation ( $H$ is the coarse mesh size parameter, and $h$ is the fine mesh size parameter, $h \ll H)$ and on the finite element space $M_{H}, M_{h}$ for the pressure approximation; the first step is to get the solution of $u_{H}, p_{H}$ on a coarse grid by the NRFE method. Generally speaking, because the grid is coarse, it does not take much time. The second step is to solve a linear equation using known $u_{H}, p_{H}$. In some papers, the third step is to solve a correction problem on the coarse grid by the correction technique. In recent years, the two-layer grid method has attracted more and more attention. Layton [9], $\mathrm{Li}$ and Hou [10], He and Li [11], Mei [13], Shi and Wang [14], Chen [15], and others have done a lot of work in this field.

The other main work of this paper is to prove the stability and convergence rate of the TNRFE method for solving Navier-Stokes equations. The design process and numerical experiment of the algorithm are given.

The TNRFE method is used to solve a nonlinear problem on a coarse grid with a grid size of $H$ and a linear problem on a fine grid with a grid size of $h$. However, the NRFE method is used to solve a nonlinear problem on a fine grid with a grid size of $h$. Therefore, the TNRFE method is simpler than the NRFE method in calculation and can save a lot of CPU time, and the TNRFE method has the same order of convergence speed as the NRFE method when the grid size is selected properly. In this paper, the numerical experiments and theoretical analysis prove that the Newton TNRFE method and the NRFE method have the same order of convergence speed under the condition of $h=O\left(H^{2}\right)$.

In Section 2, we briefly review some knowledge and important properties of Sobolev spaces and give the variational forms and the existence, uniqueness, and regularity estimates of solutions for the Navier-Stokes equations. In Section 3, we first introduce the construction and properties of the nonconforming rectangular element space. We explain the NRFE method, existence, uniqueness, and convergence rate of the approximation solution for Stokes equations. The design process and finite element analysis of the algorithm are given in detail. In Section 4, the NRFE method, existence, uniqueness, and convergence rate of the approximate solution for Navier-Stokes equations are discussed. In Section 5, the stability and convergence rate of the solution of the TNRFE method for Navier-Stokes equations are proved. In Section 6, a large number of numerical experiments are given to verify the correctness and effectiveness of the algorithm.

\section{Governing Equations}

In this paper, we study the linearized energy-conservative finite element method for the following nonlinear Navier-Stokes equations:

$$
\begin{cases}-v \Delta u+(u \cdot \nabla) u+\nabla p=f, & \forall x \in \Omega, \\ \nabla \cdot u=0, & \forall x \in \Omega, \\ u=0, & \forall x \in \Gamma,\end{cases}
$$

where $\Omega$ is a bounded domain and convex domain in $\mathrm{R}^{d}$ $(d=2,3), \quad u=\left(u_{1}(x), u_{2}(x)\right)$ is the velocity vector, $p=p(x)$ is the pressure, $f=f(x)$ is the external force density, and $v>0$ is the dynamic viscosity coefficient. 
In order to describe the variational form of NavierStokes equations, the Sobolev space is introduced:

$$
\begin{aligned}
X & =H_{0}^{1}(\Omega)^{2}, \\
Y & =L^{2}(\Omega)^{2}, \\
M & =L_{0}^{2}(\Omega)=\left\{q \in L^{2}(\Omega) ; \int_{\Omega} q \mathrm{~d} x=0\right\},
\end{aligned}
$$

where $(\cdot, \cdot),\|\cdot\|_{0}$ denotes the inner product and norm on $L^{2}(\Omega)^{d}$ and $X$ is equipped with the usual inner product and norm.

$$
\begin{aligned}
((u, v)) & =(\nabla u, \nabla v), \\
\|\nabla u\|_{0} & =((u, u))^{1 / 2},
\end{aligned}
$$

and $\langle\cdot, \cdot\rangle_{\Gamma},\left\||\cdot \||_{\Gamma}\right.$ denotes the inner product and norm over $L^{2}(\Gamma)^{d}$.

Define, respectively, the continuous bilinear form on $X \times X$ and $X \times M$ :

$$
\begin{aligned}
& a(u, v)=v((u, v)), \quad \forall u, v \in X, \\
& d(v, q)=(\nabla \cdot v, q), \quad \forall v \in X, q \in M .
\end{aligned}
$$

In addition, we introduce a closed subset of $X$ :

$$
V=\{v \in X ; d(v, q)=0, \forall q \in M\} .
$$

Let $A u=-\Delta u$. As we all know, $A$ is a linear, unbounded, and self-conjugate operator in $Y$, defined in

$$
D(A)=H^{2}(\Omega)^{2} \cap X .
$$

Theorem 1 (see [4]). Let $\Omega$ be a smooth or convex polygon of $C^{2}$; for given $f \in Y$, the Stokes problem

$$
\begin{cases}-v \Delta u+\nabla p=f, & \forall x \in \Omega, \\ \nabla \cdot u=0, & \forall x \in \Omega, \\ u=0, & \forall x \in \Gamma,\end{cases}
$$

there exists a unique solution of $(u, p) \in D(A) \times\left(H^{1}(\Omega) \cap M\right)$ and satisfies

$$
\|u\|_{2}+\|p\|_{1} \leq c\|f\|_{0},
$$

where $c$ is a positive constant dependent on $\Omega$ and $\|\cdot\|_{i}$ is a norm over $H^{i}(\Omega)$ or $H^{i}(\Omega)^{2}(i=1,2)$.

Define the trilinear form on $X \times X \times X$ :

$$
\begin{aligned}
b(u, v, w) & =((u \cdot \nabla) v, w)+\frac{1}{2}((\nabla \cdot u) v, w) \\
& =\frac{1}{2}((u \cdot \nabla) v, w)-\frac{1}{2}((u \cdot \nabla) w, v), \quad \forall u, v, w \in X,
\end{aligned}
$$

which satisfies the following properties:

$$
\begin{gathered}
((u \cdot \nabla) v, w)+((u \cdot \nabla) w, v)=\int_{\Gamma}(w \cdot v)(u \cdot n) \mathrm{d} s-\int_{\Omega}(w \cdot v) \nabla \cdot u \mathrm{~d} x, \forall u, v, w \in X \\
|b(u, v, w)| \leq C_{b}\|\nabla u\|_{0}\|\nabla v\|_{0}\|\nabla w\|_{0}, \forall u, v, w \in X \\
b(u, v, w)=-b(u, w, v), \forall u, v, w \in X .
\end{gathered}
$$

So, the variational form of problem (1) is

$$
\begin{cases}\text { find }(u, p) \in X \times M, & \text { so } \\ a(u, v)-d(v, p)+b(u, u, v)=(f, v), & \forall v \in X, \\ d(u, q)=0, & \forall q \in M,\end{cases}
$$

and problem (2) in the variational form is

$$
\begin{cases}\text { find }(u, p) \in X \times M, & \text { so, } \\ a(u, v)-d(v, p)=(f, v), & \forall v \in X, \\ d(u, q)=0, & \forall q \in M .\end{cases}
$$

The inequalities that will be used in this article are described here first.
(1) Hölder's inequality:

If $u \in L^{p}(\Omega)$ and $v \in L^{q}(\Omega), p, q$ are positive real numbers, then

$$
\int_{\Omega} u v \mathrm{~d} x\|u\|_{L^{p}}\|v\|_{L^{q}}, \frac{1}{p}+\frac{1}{q}=1 .
$$

(2) Young's inequality:

If $a, b, p, q$, and $\epsilon$ are all positive real numbers, then

$$
a b \leq \frac{\epsilon a^{p}}{p}+\frac{\epsilon^{-q / p} b^{q}}{q}, \frac{1}{p}+\frac{1}{q}=1 .
$$

(3) Sobolev's embedding theorem: 
Let $m>0$ be an integer, $1 \leq p<\infty$, and let $\Omega$ be an open subset of $R^{n}$ and have Lipschitz continuous boundary, so the following embedding relationship holds:

$$
W^{m, p}(\Omega) \longrightarrow \begin{cases}L^{q}(\Omega), & q=\frac{n p}{n-m p}, \quad m p<n \\ L^{q}(\Omega), & \forall q \in[1, \infty), \quad m p=n \\ C^{0, m-n / p}(\bar{\Omega}), & m p>n>(m-1) p \\ C^{0, \alpha}(\bar{\Omega}), & \forall \alpha \in(0,1), \quad n=(m-1) p \\ C^{0,1}(\bar{\Omega}), & n<(m-1) p\end{cases}
$$

In this paper, we give the existence and uniqueness of solutions for the classical Navier-Stokes equations.

Theorem 2 (see [16]). If $v$ and $f \in X^{\prime}$ satisfy

$$
\frac{C_{b}}{v^{2}}\|f\|_{-1}<1,\|f\|_{-1}=\sup _{v \in X} \frac{(f, v)}{\|\nabla v\|_{0}},
$$

then problem (11) has a unique solution of $(u, p) \in D(A) \times\left(H^{1}(\Omega) \cap M\right)$, and $u \in V$ satisfies

$$
\|\nabla u\|_{0} \leq \frac{1}{v}\|f\|_{-1}
$$

Theorem 3 (see [12]). If $f \in Y$ satisfies uniqueness condition (8), then the solution of problem (11) satisfies the following regularity estimate:

$$
\|u\|_{2}+\|p\|_{1} \leq C
$$

where $C$ is a positive constant dependent on $(\nu, f, \Omega)$.

\section{A Nonconforming Rotated Finite Element Method for the Stokes Equations}

In the first section of this section, we review the construction and properties of the nonconforming rotated rectangular element space. In the second section, we discuss the existence, uniqueness, and stability of the solution of the problem approximated by the NRFE method. In the third section, we give the convergence rate of this method. In the fourth section, the concrete design of the algorithm is introduced. The key of solving the Stokes equations with the NRFE method is to condense the internal degree of freedom in the process of establishing the stiffness. In the fifth section, we describe the finite element analysis of the method.
In this paper, we use a rectangular partition of the same scale for the velocity and pressure. Here and later, $h$ is a positive real parameter that tends to zero, and $0<h \leq 1$.

Definition 1. Let $\tau_{h}=\tau_{h}(\Omega)$ be a partition of the region $\bar{\Omega}$ and $\Omega_{j}$ be any unit of $\tau_{h}$; if $\tau_{h}$ satisfies the following conditions:

(i) There exists a constant $\sigma>0$ so that $h_{j} / \rho_{j} \leq \sigma, \forall \Omega_{j} \in \tau_{h}, \quad$ where $h_{j}=\operatorname{diam}\left(\Omega_{j}\right)=\sup \left\{|x-y|, \forall x, y \in \Omega_{j}\right\}$ and $\rho_{j}=$ $\sup \left\{\operatorname{diam}(S), S\right.$ is any inside ball of $\left.\Omega_{j}\right\}$

(ii) There exists a constant $\gamma>0$ that has nothing to do with $h$ such that $\max \left\{h / h_{j}, \forall \Omega_{j} \in \tau_{h}\right\} \leq \gamma$, where $h=\max _{\Omega_{j}}\left\{h_{j}\right\}$

then $\tau_{h}$ is called the quasi-uniform regular partition of the region $\bar{\Omega}$.

Let $\bar{\Omega}=\cup_{j=1}^{J} \bar{\Omega}_{j} \subset R^{2}$, and $\Omega_{j}$ is any quasi-uniform regular quadrilateral element of the region $\bar{\Omega}$, recorded as

$$
\Gamma_{j}=\Gamma \cap \Gamma_{j}, \Gamma_{j k}=\Gamma_{k j}=\partial \Omega_{j} \cap \Gamma_{k} .
$$

The midpoints of $\Gamma_{j}$ and $\Gamma_{j k}$ are, respectively, $\xi_{j}$ and $\xi_{j k}$. We define the standard base on the standard unit:

$$
\begin{aligned}
& Q(\widehat{K})=\operatorname{Span}\left\{1, \xi, \eta, \xi \eta,\left(\xi^{2}-\frac{5}{3} \xi^{4}\right)-\left(\eta^{2}-\frac{5}{3} \eta^{4}\right)\right\}, \\
& \widehat{Q}\left(\Omega_{j}\right)=\left\{v: v=\widehat{v}^{\circ} \mathrm{F}_{\mathrm{j}}^{-1}, \widehat{\mathrm{v}} \in \mathbb{Q}(\widehat{\mathrm{K}})\right\},
\end{aligned}
$$

where $F_{j}$ is a reversible affine transformation and $F_{j}(\widehat{K})=\Omega_{j}$.

Now, we construct the finite element space.

(1) Nonconforming rectangular finite element space

$$
X_{h}=\left\{v: v_{j}=\left.v\right|_{\Omega_{j}} \in \mathbb{Q}\left(\Omega_{j}\right) \times Q \mathcal{Q}\left(\Omega_{j}\right), v_{j}\left(\xi_{j k}\right)=v_{k}\left(\xi_{k j}\right), v_{j}\left(\xi_{j}\right)=0, \forall j, k\right\}
$$


satisfies the following properties:

(i) Inverse inequality:

$$
\|\nabla \chi\|_{0, j} \leq c h_{j}^{-1}\|\chi\|_{0, j}, \forall \chi \in X_{h} .
$$

(ii) Poincaré-Friedrichs inequality:

$\|v\|_{0, j}^{2} \leq h^{2}\|\nabla v\|_{0, j}^{2}+\left(\int_{\Gamma_{1}} v \mathrm{~d} s\right)^{2}, \forall v \in H^{1}\left(\Omega_{j}\right)^{2}, \Gamma_{1} \subseteq \partial \Omega_{j}$.

(iii) Trace inequality:

$\|v\| \|_{j} \leq c\left\{h_{j}^{-1 / 2}\|v\|_{0, j}+\|v\|_{0, j}^{1 / 2}\|\nabla v\|_{0, j}^{1 / 2}\right\}, \forall v \in H^{1}\left(\Omega_{j}\right) .^{2}$

(iv)

$$
\int_{\Gamma_{j k}}\left[v_{i}\right] \mathrm{d} s=0, \int_{\Gamma_{j}} v_{i} \mathrm{~d} s=0, \forall v=\left(v_{1}, v_{2}\right)^{T} \in X_{h}, i=1,2,
$$

where $\|\cdot\|_{m, j}^{2}=\|\cdot\|_{H^{m}}\left(\Omega_{j}\right)^{2},\|\cdot\|_{j}^{2}=\sum_{k \in Z_{j}}$ $\|\cdot\|_{L^{2}\left(\Gamma_{j k}\right)^{2}}^{2}$, and $Z_{j}=\left\{k, \bar{\Omega}_{k} \cap \bar{\Omega}_{j} \neq \varnothing\right\} . \Gamma_{j k}$ can be replaced by $\Gamma_{j}$ at the outer boundary, and $\left[v_{i}\right]=\left.v_{i}\right|_{\Gamma_{j k}}-\left.v_{i}\right|_{\Gamma_{k j}}$ means the jump of $v_{i}$ on the public boundary $\Gamma_{j k}, 1 \leq j \leq J$.

(2) Piecewise constant finite element space

$$
M_{h}=\left\{q \in M:\left.q\right|_{\Omega_{j}} \in \mathrm{P}_{0}\left(\Omega_{j}\right), \forall j\right\}
$$

satisfies the following properties:

(i) Inverse inequality:

$$
\|\nabla q\|_{0, j} \leq c h_{j}^{-1}\|q\|_{0, j}, \forall q \in M_{h} .
$$

(ii) Poincaré-Friedrichs inequality:

$$
\|p\|_{0, j}^{2} \leq h^{2}\|\nabla p\|_{0, j}^{2}+\left(\int_{\Gamma_{1}} p \mathrm{~d} s\right)^{2}, \forall p \in H^{1}\left(\Omega_{j}\right), \Gamma_{1} \subseteq \partial \Omega_{j} .
$$

(iii) Trace inequality:

$$
\|\mid p\| \|_{j} \leq c\left\{h_{j}^{-1 / 2}\|p\|_{0, j}+\|p\|_{0, j}^{1 / 2}\|\nabla p\|_{0, j}^{1 / 2}\right\}, \forall p \in H^{1}\left(\Omega_{j}\right),
$$

where $\quad\|\cdot\|_{m, j}^{2}=\|\cdot\|_{H^{m}\left(\Omega_{j}\right)}^{2}, \quad\|\cdot\|_{j}^{2}=\sum_{k \in Z_{j}} \|$ $\cdot \|_{L^{2}\left(\Gamma_{j k}\right)}^{2}$, and $Z_{j}=\left\{k, \bar{\Omega}_{k} \cap \bar{\Omega}_{j} \neq \varnothing\right\} . \Gamma_{j k}$ can be replaced by $\Gamma_{j}$ at the outer boundary, $1 \leq j \leq J$. form

Let $(\cdot, \cdot)_{j}=(\cdot, \cdot)_{\Omega_{j}}, 1 \leq j \leq J$, define the discrete bilinear

$$
\begin{aligned}
& a_{h}(u, v)=v \sum_{j=1}^{J}(\nabla u, \nabla v)_{j}, \forall u, v \in X \cup X_{h} \\
& d_{h}(v, q)=\sum_{j=1}^{J}(\nabla \cdot v, q)_{j}, \forall v \in X_{h}, q \in M_{h},
\end{aligned}
$$

and norm

$$
\|v\|_{1, h}=\left(\sum_{j=1}^{J}\|\nabla v\|_{0, j}^{2}\right)^{1 / 2}=\sqrt{\frac{1}{v} a_{h}(v, v)}, \forall v \in X_{h} .
$$

In addition, we introduce a closed subset of $X_{h}$ :

$$
V_{h}=\left\{v \in X_{h} ; d_{h}(v, q)=0, \forall q \in M_{h}\right\}
$$

Define operator $\pi_{j}: H^{1}\left(\Omega_{j}\right) \longrightarrow Q\left(\Omega_{j}\right)$, which satisfies

$$
\int_{e_{j}^{i}} \pi_{j} v \mathrm{~d} s=\int_{e_{j}^{i}} v \mathrm{~d} s, \forall v \in H^{1}\left(\Omega_{j}\right), i=1,2,3,4,
$$

and is equivalent to

$$
\pi_{j} v\left(a_{j}^{i}\right)=\frac{1}{\left|e_{j}^{i}\right|} \int_{e_{j}^{i}} v \mathrm{~d} s, i=1,2,3,4,
$$

where $e_{j}^{i}(i=1,2,3,4)$ are the four edges of $\Omega_{j}$, and their corresponding midpoints are $a_{j}^{i}(i=1,2,3,4)$, satisfying the following properties:

(i)

$$
\left\|\nabla\left(v-\pi_{j} v\right)\right\|_{0, j} \leq c\|\nabla v\|_{0, j}, \forall v \in H^{1}\left(\Omega_{j}\right) .
$$

(ii)

$$
\left\|\nabla\left(\pi_{j} v\right)\right\|_{0, j} \leq c\|\nabla v\|_{0, j}, \forall v \in H^{1}\left(\Omega_{j}\right) .
$$

Define operator $\Pi_{h}: X \longrightarrow X_{h}$, which satisfies

$$
\left.\left(\Pi_{h} v\right)_{i}\right|_{\Omega_{j}}=\pi_{j} v_{i}, \quad \forall v \in X, \forall j, i=1,2,
$$

and satisfies the following properties:

$$
d_{h}\left(\Pi_{h} v-v, q\right)=0, \forall q \in M_{h} .
$$

(ii)

$$
\left\|\Pi_{h} v\right\|_{1, h} \leq c\|\nabla v\|_{0}, \quad \forall v \in X
$$

Define the projection operator $R_{h}: H^{2}(\Omega)^{2} \longrightarrow X_{h}$, which satisfies

$$
R_{h} v(\xi)=v(\xi), \forall \xi=\xi_{j k} \text { or } \xi_{j}
$$

and $P_{0}: H^{1}(\Omega)^{2} \longrightarrow \Lambda_{h} \times \Lambda_{h}$ satisfies

$$
\left\langle P_{0} v_{j}, z\right\rangle_{\Gamma}=\left\langle\frac{\partial v_{j}}{\partial n_{j}}, z\right\rangle_{\Gamma}, \forall z \in \mathscr{P}_{0}(\Gamma)^{2}, \forall \Gamma=\Gamma_{j k} \text { or } \Gamma_{j},
$$

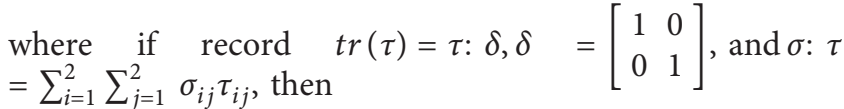




$$
\Lambda_{h}=\left\{\lambda: \lambda_{j k}=\operatorname{tr}_{\Gamma_{j k}}\left(\left.\lambda\right|_{\Omega_{j}}\right) \in \mathrm{P}_{0}\left(\Gamma_{j k}\right) ; \lambda_{j k}+\lambda_{k j}=0 ; \lambda_{j}=\operatorname{tr}_{\Gamma_{j}}\left(\left.\lambda\right|_{\Omega_{j}}\right) \in \mathrm{P}_{0}\left(\Gamma_{j}\right)\right\} .
$$

Define the projection operator $S_{h}: H^{1}(\Omega) \longrightarrow M_{h}$, which satisfies

$$
\left(S_{h} q, z\right)=(q, z), \forall z \in M_{h},
$$

and $Q_{0}: H^{1}(\Omega) \longrightarrow \mathrm{P}_{0}(\Gamma)$ satisfies

$$
\left\langle Q_{0} q, z\right\rangle_{\Gamma}=\langle q, z\rangle_{\Gamma}, \forall z \in \mathrm{P}_{0}(\Gamma), \forall \Gamma=\Gamma_{j k} \mathrm{or} \Gamma_{j} .
$$

They satisfy the following properties:

(i) Orthogonality:

$$
\left\langle P_{0} v_{j}, w_{j}\right\rangle_{\Gamma_{j k}}+\left\langle P_{0} v_{k}, w_{k}\right\rangle_{\Gamma_{k j}}=\left\langle P_{0} v_{j}, w_{j}-w_{k}\right\rangle_{\Gamma_{j k}}=0, \forall w \in X_{h} .
$$

(ii) Approximation property:

$$
\begin{aligned}
& \left\|v-R_{h} v\right\|_{0}+h\left(\sum_{j=1}^{J}\left\|\nabla\left(v-R_{h} v\right)\right\|_{0, j}^{2}\right)^{1 / 2}+h^{1 / 2}\left(\sum_{j=1}^{J}\left\|\mid v-R_{h} v\right\|_{j}^{2}\right)^{1 / 2} \\
& \quad+h^{3 / 2}\left(\sum_{j=1}^{J}\left\|\left|\frac{\partial v}{\partial n_{j}}-P_{0} v \|\right|_{j}^{2}\right)^{1 / 2}\right. \\
& \leq c h^{2}\|v\|_{2}, v \in H^{2}(\Omega)^{2} \\
& \left\|q-S_{h} q\right\|_{0}+h^{1 / 2}\left(\sum_{j=1}^{J}\left\|q-Q_{0} q\right\|_{j}^{2}\right)^{1 / 2} \leq c h\|q\|_{1}, q \in H^{1}(\Omega) .
\end{aligned}
$$

Here and later, $n_{j}, 1 \leq j \leq J$, represents the unit outer normal vector of $\partial \Omega_{j}$.

Then, the nonconforming finite element approximation problem of (12) is

$$
\begin{cases}\text { find }\left(u_{h}, p_{h}\right) \in X_{h} \times M_{h}, & \text { so } \\ a_{h}\left(u_{h}, v\right)-d_{h}\left(v, p_{h}\right)=(f, v), & \forall v \in X_{h}, \\ d_{h}\left(u_{h}, q\right)=0, & \forall q \in M_{h} .\end{cases}
$$

Proposition 1 (see [7]). If the bilinear form $d(\cdot, \cdot)$ satisfies the inf - sup condition, there is a constant $\beta>0$ such that

$$
\sup _{v \in X} \frac{d(v, q)}{\|\nabla v\|_{0}} \geq \beta\|q\|_{0}, \forall q \in M,
$$

then the bilinear form $d_{h}(\cdot, \cdot)$ satisfies the discrete inf - sup condition: there is a positive constant $\beta_{0}$ such that

$$
\sup _{v \in X_{h}} \frac{d_{h}(v, q)}{\|v\|_{1, h}} \geq \beta_{0}\|q\|_{0}, \forall q \in M_{h} .
$$

Theorem 4 (see [7]). Under the condition of Proposition 1, if $V_{h}$ is not empty, problem (48) has a unique solution $\left(u_{h}, p_{h}\right) \in X_{h} \times M_{h}$, and $u_{h} \in V_{h}$ satisfies

$$
\left\|u_{h}\right\|_{1, h} \leq \frac{1}{v}\|f\|_{*},\|f\|_{*}=\sup _{v \in X_{h}} \frac{(f, v)}{\|v\|_{1, h}} .
$$

Proposition 2 (see [7]). Let $(u, p)$ and $\left(u_{h}, p_{h}\right)$ be, respectively, the solutions of problems (12) and (48); there is a positive constant of $c$ such that

$$
\begin{aligned}
& \left\|u-u_{h}\right\|_{1, h} \leq c\left(\inf _{v \in X_{h}}\|u-v\|_{1, h}+\sup _{v \in V_{h}} \frac{\left|a_{h}(u, v)-(f, v)\right|}{\|v\|_{1, h}}\right) \\
& \left\|p-p_{h}\right\|_{0} \leq c\left(\inf _{q \in M_{h}}\|p-q\|_{0}+\sup _{v \in X_{h}} \frac{\left|a_{h}(u, v)-d_{h}(v, p)-(f, v)\right|}{\|v\|_{1, h}}+\inf _{v \in X_{h}}\|u-v\|_{1, h}+\sup _{v \in V_{h}} \frac{\left|a_{h}(u, v)-(f, v)\right|}{\|v\|_{1, h}}\right) .
\end{aligned}
$$


Proposition 3 (see [7]). Let $\langle f, g\rangle_{j}=\int_{\partial \Omega_{j}} f g d s$, for $\forall \phi, w \in X \cup X_{h}$, satisfy the following properties:

$$
\begin{gathered}
\left|\sum_{j=1}^{J}\left\langle\frac{\partial w}{\partial n_{j}}, \phi\right\rangle_{j}\right| \leq c h\|w\|_{2}\|\phi\|_{1, h}, \forall w \in D(A) \\
\left|\sum_{j=1}^{J}\left\langle q, \phi \cdot n_{j}\right\rangle_{j}\right| \leq c h\|q\|_{1}\|\phi\|_{1, h}, \forall q \in H^{1}(\Omega) .
\end{gathered}
$$

Theorem 5 (see [7]). Let $(u, p) \in H^{2}(\Omega)^{2} \times H^{1}(\Omega)$ and $\left(u_{h}, p_{h}\right) \in X_{h} \times M_{h}$ be, respectively, the solutions of problems (12) and (48); then,

$$
\left\|u-u_{h}\right\|_{1, h}+\left\|p-p_{h}\right\|_{0} \leq \operatorname{ch}\left(\|u\|_{2}+\|p\|_{1}\right) .
$$

Using the dual argument method, we estimate the error of $L^{2}$.

Consider the linear duality problem:

$$
\begin{cases}-\nu \Delta \psi+\nabla \chi=u-u_{h} & \forall x \in \Omega, \\ \nabla \cdot \psi=0 & \forall x \in \Omega, \\ \psi=0 & \forall x \in \Gamma .\end{cases}
$$

The variational form is

$$
\begin{cases}\text { find }(\psi, \chi) \in X \times M, & \text { so } \\ a(v, \psi)-d(v, \chi)=\left(u-u_{h}, v\right), & \forall v \in X, \\ d(\psi, q)=0, & \forall q \in M .\end{cases}
$$

If $u$ is the nonsingular solution of problem (2), then problem (56) has a unique solution (see [16]).

In order to establish an estimate of the $L^{2}$ error of velocity, Aubin-Nitsche argumentation method is used here. Let problem (34) be $H^{2}$ regular, i.e.,

$$
\|\psi\|_{2}+\|\chi\|_{1} \leq c\left\|u-u_{h}\right\|_{0} .
$$

Let $(\psi, \chi)$ be the solution of problem (56), and $\left(\psi_{h}, \chi_{h}\right) \in X_{h} \times M_{h}$, satisfying

$$
\left\|\psi-\psi_{h}\right\|_{1, h}+\left\|\chi-\chi_{h}\right\|_{0} \leq \operatorname{ch}\left(\|\psi\|_{2}+\|\chi\|_{1}\right) .
$$

Theorem 6 (see [7]). Let $(u, p) \in H^{2}(\Omega)^{2} \times H^{1}(\Omega)$ and $\left(u_{h}, p_{h}\right) \in X_{h} \times M_{h}$ be, respectively, the solutions of problems (12) and (48); if problem (55) is $\mathrm{H}^{2}$ regular, then

$$
\left\|u-u_{h}\right\|_{0} \leq c h^{2}\left(\|u\|_{2}+\|p\|_{1}\right) .
$$

This section presents the algorithm design for solving problem (48).

3.1. Step 1: Condensation of Internal Degrees of Freedom. Let the four edges of the standard cell $\widehat{K}=[-1,1]^{2}$ be, respectively, $\widehat{e}_{1}, \widehat{e}_{2}, \widehat{e}_{3}$, and $\widehat{e}_{4}$; the corresponding midpoints are $\widehat{a}_{1}=(0,-1), \widehat{a}_{2}=(1,0), \widehat{a}_{3}=(0,1)$, and $\widehat{a}_{4}=(-1,0)$, and the shape functions for each node of the Douglas rotation element obtained are, respectively,

$$
\begin{aligned}
& \varphi_{1}(\xi, \eta)=\frac{1}{4}-\frac{1}{2} \eta+\frac{3}{8}\left(\left(\xi^{2}-\frac{5}{3} \xi^{4}\right)-\left(\eta^{2}-\frac{5}{3} \eta^{4}\right)\right) \\
& \varphi_{2}(\xi, \eta)=\frac{1}{4}+\frac{1}{2} \xi-\frac{3}{8}\left(\left(\xi^{2}-\frac{5}{3} \xi^{4}\right)-\left(\eta^{2}-\frac{5}{3} \eta^{4}\right)\right) \\
& \varphi_{3}(\xi, \eta)=\frac{1}{4}+\frac{1}{2} \eta+\frac{3}{8}\left(\left(\xi^{2}-\frac{5}{3} \xi^{4}\right)-\left(\eta^{2}-\frac{5}{3} \eta^{4}\right)\right) \\
& \varphi_{4}(\xi, \eta)=\frac{1}{4}-\frac{1}{2} \xi-\frac{3}{8}\left(\left(\xi^{2}-\frac{5}{3} \xi^{4}\right)-\left(\eta^{2}-\frac{5}{3} \eta^{4}\right)\right)
\end{aligned}
$$

It is easy to verify

$$
\int_{\widehat{e}_{i}} \varphi_{j} \mathrm{~d} \widehat{s}=\delta_{i j}\left|\widehat{e}_{i}\right|, i, j=1,2,3,4,
$$

where $\delta_{i j}$ is the Kronecker symbol and $\left|\widehat{e}_{i}\right|$ is the length of the edge of $\widehat{e}_{i}$.

The element speed interpolation with additional nodefree speed terms can be expressed as follows:

$$
\left\{\begin{array}{l}
u_{1}=\sum_{i=1}^{4} \varphi_{i} u_{i}^{1}+\lambda_{1} \xi \eta, \\
u_{2}=\sum_{i=1}^{4} \varphi_{i} u_{i}^{2}+\lambda_{2} \xi \eta,
\end{array}\right.
$$

where $\lambda_{1}$ and $\lambda_{2}$ are the undetermined coefficient in the element's internal definition, known as the internal degree of freedom.

The expression is expressed in the matrix form:

$$
u=u_{a}+u_{\lambda}
$$

i.e.,

$$
\begin{aligned}
{\left[\begin{array}{l}
u_{1} \\
u_{2}
\end{array}\right]=} & {\left[\begin{array}{cccccccc}
\varphi_{1} & \varphi_{2} & \varphi_{3} & \varphi_{4} & 0 & 0 & 0 & 0 \\
0 & 0 & 0 & 0 & \varphi_{1} & \varphi_{2} & \varphi_{3} & \varphi_{4}
\end{array}\right]\left[\begin{array}{l}
u_{1}^{1} \\
u_{2}^{1} \\
u_{3}^{1} \\
u_{4}^{1} \\
u_{1}^{2} \\
u_{2}^{2} \\
u_{3}^{2} \\
u_{4}^{2}
\end{array}\right] } \\
& +\left[\begin{array}{cc}
\xi_{\eta} & 0 \\
0 & \xi \eta
\end{array}\right]\left[\begin{array}{l}
\lambda_{1} \\
\lambda_{2}
\end{array}\right]
\end{aligned}
$$

The element pressure is interpolated as follows:

$$
p=p_{1} \zeta_{1},
$$

where $\zeta_{1}=1$.

We discretize equation (48) on the unit $\Omega_{j}$ using the interpolation functions of $u$ and $p$. Since $v=\left(v_{1}, v_{2}\right)^{T} \in X_{h}$ and $q \in M_{h}$ is arbitrary, we can choose the following: 
(1) $v=\left[\begin{array}{c}\varphi_{l} \\ 0\end{array}\right], l=1,2,3,4$, and $v=\left[\begin{array}{c}\xi \eta \\ 0\end{array}\right]$.

$$
\begin{aligned}
& v(\nabla u, \nabla v)_{j} \\
& =v \iint_{\Omega_{j}}\left(\nabla u_{1} \cdot \nabla v_{1}+\nabla u_{2} \cdot \nabla v_{2}\right) \mathrm{d} \Omega \\
& =\left\{\begin{array}{cc}
\nu \sum_{j=1}^{4} u_{j}^{1} \iint_{\Omega_{j}} \nabla \varphi_{j} \nabla \varphi_{l} \mathrm{~d} \Omega+\nu \lambda_{1} \iint_{\Omega_{j}} \nabla(\xi \eta) \nabla \varphi_{l} \mathrm{~d} \Omega & v_{1}=\varphi_{l}, l=1,2,3,4, \\
\nu \sum_{j=1}^{4} u_{j}^{1} \iint_{\Omega_{j}} \nabla \varphi_{j} \nabla(\xi \eta) \mathrm{d} \Omega+\nu \lambda_{1} \iint_{\Omega_{j}} \nabla(\xi \eta) \nabla(\xi \eta) \mathrm{d} \Omega & v_{1}=\xi \eta,
\end{array}\right\} \\
& (\nabla \cdot v, p)_{j} \\
& =\iint_{\Omega_{j}} p\left(\frac{\partial v_{1}}{\partial x_{1}}+\frac{\partial v_{2}}{\partial x_{2}}\right) \mathrm{d} \Omega \\
& = \begin{cases}p_{1} \iint_{\Omega_{j}} \zeta_{1} \frac{\partial \varphi_{l}}{\partial x_{1}} \mathrm{~d} \Omega & v_{1}=\varphi_{l}, l=1,2,3,4, \\
p_{1} \iint_{\Omega_{j}} \zeta_{1} \frac{\partial(\xi \eta)}{\partial x_{1}} \mathrm{~d} \Omega & v_{1}=\xi \eta,\end{cases} \\
& (f, v)_{j}=\iint_{\Omega_{j}}\left(f_{1} v_{1}+f_{2} v_{2}\right) \mathrm{d} \Omega \\
& = \begin{cases}\iint_{\Omega_{j}} f_{1} \varphi_{l} \mathrm{~d} \Omega & v_{1}=\varphi_{l}, l=1,2,3,4, \\
\iint_{\Omega_{j}} f_{1} \xi \eta \mathrm{d} \Omega & v_{1}=\xi \eta .\end{cases}
\end{aligned}
$$

(2) $v=\left[\begin{array}{c}0 \\ \varphi_{l}\end{array}\right], l=1,2,3,4$, and $v=\left[\begin{array}{c}0 \\ \xi_{\eta}\end{array}\right]$.

$$
\begin{aligned}
& v(\nabla u, \nabla v)_{j}= \begin{cases}v \sum_{j=1}^{4} u_{j}^{2} \iint_{\Omega_{j}} \nabla \varphi_{j} \nabla \varphi_{l} \mathrm{~d} \Omega+v \lambda_{1} \iint_{\Omega_{j}} \nabla(\xi \eta) \nabla \varphi_{l} \mathrm{~d} \Omega & v_{2}=\varphi_{l}, l=1,2,3,4, \\
v \sum_{j=1}^{4} u_{j}^{2} \iint_{\Omega_{j}} \nabla \varphi_{j} \nabla(\xi \eta) \mathrm{d} \Omega+v \lambda_{1} \iint_{\Omega_{j}} \nabla(\xi \eta) \nabla(\xi \eta) \mathrm{d} \Omega & v_{2}=\xi \eta,\end{cases} \\
&(\nabla \cdot v, p)_{j}= \begin{cases}p_{1} \iint_{\Omega_{j}} \zeta_{1} \frac{\partial \varphi_{l}}{\partial x_{2}} \mathrm{~d} \Omega & v_{2}=\varphi_{l}, l=1,2,3,4, \\
p_{1} \iint_{\Omega_{j}} \zeta_{1} \frac{\partial(\xi \eta)}{\partial x_{2}} \mathrm{~d} \Omega & v_{2}=\xi \eta,\end{cases} \\
&(f, v)_{j}=\left\{\begin{array}{l}
\iint_{\Omega_{j}} f_{2} \varphi_{l} \mathrm{~d} \Omega \quad v_{2}=\varphi_{l}, l=1,2,3,4, \\
\iint_{\Omega_{j}} f_{2} \xi \eta \mathrm{d} \Omega \quad v_{2}=\xi \eta .
\end{array}\right.
\end{aligned}
$$


(3) $q=\zeta_{1}$.

$$
(\nabla \cdot u, q)_{j}=\sum_{j=1}^{4} u_{j}^{1} \iint_{\Omega_{j}} \frac{\partial \varphi_{j}}{\partial x_{1}} \zeta_{1} \mathrm{~d} \Omega+\lambda_{1} \iint_{\Omega_{j}} \frac{\partial(\xi \eta)}{\partial x_{1}} \zeta_{1} \mathrm{~d} \Omega+\sum_{j=1}^{4} u_{j}^{2} \iint_{\Omega_{j}} \frac{\partial \varphi_{j}}{\partial x_{2}} \zeta_{1} \mathrm{~d} \Omega+\lambda_{2} \iint_{\Omega_{j}} \frac{\partial(\xi \eta)}{\partial x_{2}} \zeta_{1} \mathrm{~d} \Omega
$$

By synthesizing (1)-(3) and making some adjustments, the element discrete equations are obtained:

$$
\left\{\begin{array}{r}
\sum_{i=1}^{4} K_{j i}^{11} u_{i}^{1}+\sum_{i=1}^{4} K_{j i}^{12} u_{i}^{2}+K_{j 1}^{13} \lambda_{1}+K_{j 2}^{14} \lambda_{2}+K_{j 1}^{15} p_{1}=R_{j}^{1} \\
\sum_{i=1}^{4} K_{j i}^{21} u_{i}^{1}+\sum_{i=1}^{4} K_{j i}^{22} u_{i}^{2}+K_{j 1}^{23} \lambda_{1}+K_{j 2}^{24} \lambda_{2}+K_{j 1}^{25} p_{1}=R_{j}^{2} \\
\sum_{i=1}^{4} K_{1 i}^{31} u_{i}^{1}+\sum_{i=1}^{4} K_{1 i}^{32} u_{i}^{2}+K_{11}^{33} \lambda_{1}+K_{12}^{34} \lambda_{2}+K_{11}^{35} p_{1}=R_{\lambda_{1}}^{1} \\
\sum_{i=1}^{4} K_{2 i}^{41} u_{i}^{1}+\sum_{i=1}^{4} K_{2 i}^{42} u_{i}^{2}+K_{21}^{43} \lambda_{1}+K_{22}^{44} \lambda_{2}+K_{21}^{45} p_{1}=R_{\lambda_{2}}^{2} \\
\sum_{i=1}^{4} K_{1 i}^{51} u_{i}^{1}+\sum_{i=1}^{4} K_{1 i}^{52} u_{i}^{2}+K_{11}^{53} \lambda_{1}+K_{12}^{54} \lambda_{2}+K_{11}^{55} p_{1}=R_{1}^{3} \\
j=1,2, \cdots, 4 .
\end{array}\right.
$$

Let

$$
\delta_{j}^{i}= \begin{cases}1 & j=i \\ 0 & j \neq i\end{cases}
$$

where

$$
\begin{aligned}
& K_{j i}^{\tau \omega}=\delta_{\tau}^{\omega} \nu \iint_{\Omega_{j}} \nabla \varphi_{i} \nabla \varphi_{j} \mathrm{~d} \Omega \tau, \omega=1,2 \\
& K_{j 1}^{13}=K_{1 j}^{31}=K_{j 2}^{24}=K_{2 j}^{42}=\nu \iint_{\Omega_{j}} \nabla(\xi \eta) \nabla \varphi_{j} \mathrm{~d} \Omega \\
& K_{j 2}^{14}=K_{2 j}^{41}=K_{j 1}^{23}=K_{1 j}^{32}=K_{12}^{34}=K_{21}^{43}=0 \\
& K_{11}^{33}=K_{22}^{44}=\nu \iint_{\Omega_{j}} \nabla(\xi \eta) \nabla(\xi \eta) \mathrm{d} \Omega \\
& K_{j 1}^{k 5}=-\iint_{\Omega_{j}} \zeta_{1} \frac{\partial \varphi_{j}}{\partial x_{k}} \mathrm{~d} \Omega k=1,2 \\
& K_{11}^{35}=-\iint_{\Omega_{j}} \zeta_{1} \frac{\partial(\xi \eta)}{\partial x_{1}} \mathrm{~d} \Omega
\end{aligned}
$$

$$
\begin{aligned}
K_{21}^{45} & =-\iint_{\Omega_{j}} \zeta_{1} \frac{\partial(\xi \eta)}{\partial x_{2}} \mathrm{~d} \Omega \\
K_{1 i}^{5 k} & =\iint_{\Omega_{j}} \frac{\partial \varphi_{i}}{\partial x_{k}} \zeta_{1} \mathrm{~d} \Omega k=1,2 \\
K_{11}^{53} & =\iint_{\Omega_{j}} \frac{\partial((\xi \eta))}{\partial x_{1}} \zeta_{1} \mathrm{~d} \Omega \\
K_{12}^{54} & =\iint_{\Omega_{j}} \frac{\partial(\xi \eta)}{\partial x_{2}} \zeta_{1} \mathrm{~d} \Omega \\
K_{11}^{55} & =0 \\
R_{j}^{k} & =\iint_{\Omega_{j}} f_{k} \varphi_{j} \mathrm{~d} \Omega k=1,2 \\
R_{1}^{3} & =0 .
\end{aligned}
$$

Since the velocity $u_{\lambda}$ in the element does not participate in the distribution of known loads, the equivalent load for $\lambda$ is zero, i.e., $R_{\lambda_{1}}^{1}=R_{\lambda_{2}}^{2}=0$.

The algebraic equations are obtained as follows:

$$
\left[\begin{array}{ccccccccc}
K_{11}^{11} & \cdots & K_{14}^{11} & 0 & \cdots & 0 & K_{11}^{13} & 0 & K_{11}^{15} \\
\vdots & \ddots & \vdots & \vdots & \ddots & \vdots & \vdots & \vdots & \vdots \\
K_{41}^{11} & \cdots & K_{44}^{11} & 0 & \cdots & 0 & K_{41}^{13} & 0 & K_{41}^{15} \\
0 & \cdots & 0 & K_{11}^{22} & \cdots & K_{14}^{22} & 0 & K_{12}^{24} & K_{11}^{25} \\
\vdots & \ddots & \vdots & \vdots & \ddots & \vdots & \vdots & \vdots & \vdots \\
0 & \cdots & 0 & K_{41}^{22} & \cdots & K_{44}^{22} & 0 & K_{42}^{24} & K_{41}^{25} \\
K_{11}^{31} & \cdots & K_{14}^{31} & 0 & \cdots & 0 & K_{11}^{33} & 0 & K_{11}^{35} \\
0 & \cdots & 0 & K_{21}^{42} & \cdots & K_{24}^{42} & 0 & K_{22}^{44} & K_{21}^{45} \\
K_{11}^{51} & \cdots & K_{14}^{51} & K_{11}^{52} & \cdots & K_{14}^{52} & K_{11}^{53} & K_{12}^{54} & 0
\end{array}\right]\left[\begin{array}{c}
u_{1}^{1} \\
\vdots \\
u_{4}^{1} \\
u_{1}^{2} \\
\vdots \\
u_{4}^{2} \\
\lambda_{1} \\
\lambda_{2} \\
p_{1}
\end{array}\right]=\left[\begin{array}{c}
R_{1}^{1} \\
\vdots \\
R_{4}^{1} \\
R_{1}^{2} \\
\vdots \\
R_{4}^{2} \\
0 \\
0 \\
0
\end{array}\right] .
$$

Abbreviations are as follows:

$$
\left[\begin{array}{ccccc}
K^{11} & 0 & K^{13} & 0 & K^{15} \\
0 & K^{22} & 0 & K^{24} & K^{25} \\
K^{31} & 0 & K^{33} & 0 & K^{35} \\
0 & K^{42} & 0 & K^{44} & K^{45} \\
K^{51} & K^{52} & K^{53} & K^{54} & 0
\end{array}\right]\left[\begin{array}{c}
U^{1} \\
U^{2} \\
\lambda_{1} \\
\lambda_{2} \\
P
\end{array}\right]=\left[\begin{array}{c}
R^{1} \\
R^{2} \\
0 \\
0 \\
0
\end{array}\right] .
$$

For the convenience of description, we write the stiffness equation of the mixed element as follows: 


$$
\left[\begin{array}{ccc}
K_{a a} & K_{a \lambda}^{T} & K_{p a}^{T} \\
K_{a \lambda} & K_{\lambda \lambda} & K_{p \lambda}^{T} \\
K_{p a} & K_{p \lambda} & 0
\end{array}\right]\left[\begin{array}{l}
U \\
\lambda \\
P
\end{array}\right]=\left[\begin{array}{l}
R \\
0 \\
0
\end{array}\right] .
$$

By eliminating the internal degrees of freedom in the upper first formula and third formula, the condensed element equations are obtained:

$$
\left[\begin{array}{cc}
K_{a a}-K_{a \lambda}^{T} K_{\lambda \lambda}^{-1} K_{a \lambda} & K_{p a}^{T}-K_{a \lambda}^{T} K_{\lambda \lambda}^{-1} K_{p \lambda}^{T} \\
K_{p a}-K_{p \lambda} K_{\lambda \lambda}^{-1} K_{a \lambda} & -K_{p \lambda} K_{\lambda \lambda}^{-1} K_{p \lambda}^{T}
\end{array}\right]\left[\begin{array}{l}
U \\
P
\end{array}\right]=\left[\begin{array}{c}
R \\
0
\end{array}\right] .
$$

The coefficient matrix in the equation is the element stiffness matrix with an additional internal velocity term, and the right-hand term is the load column array.

3.2. Step 2: Synthesis of Total Stiffness. Let the dimension of the finite element space $X_{h}$ and $M_{h}$ be, respectively, NG1 and NG2, and the basis functions of these two spaces are, respectively, $\left\{\phi_{1}, \phi_{2}, \ldots, \phi_{N G 1}\right\}$ and $\left\{\psi_{1}, \psi_{2}, \ldots, \psi_{N G 2}\right\}$. Then, the solution of the equation can be expressed as

$$
\left(u_{h}\right)^{j}=\sum_{i=1}^{N G 1}\left(u_{h}^{i}\right)^{j} \phi_{i}, j=1,2 p_{h}=\sum_{i=1}^{N G 2} p_{h}^{i} \psi_{i} .
$$

The element stiffness matrix and the load array obtained after condensation are synthesized, respectively. In order to guarantee the uniqueness of the solution of $p_{h}$, the limit of the pressure $P_{h}$ is added as follows:

$$
\sum_{j=1}^{N G 2} p_{h}^{j} \iint_{\Omega} \psi_{j} \mathrm{~d} \Omega=0
$$

The following algebraic equations are synthesized:

$$
\left[\begin{array}{ccc}
A^{11} & A^{12} & A^{13} \\
A^{21} & A^{22} & A^{23} \\
A^{31} & A^{32} & A^{33} \\
0 & 0 & A^{43}
\end{array}\right]\left[\begin{array}{c}
\left(U_{h}\right)^{1} \\
\left(U_{h}\right)^{2} \\
P_{h}
\end{array}\right]=\left[\begin{array}{c}
F^{1} \\
F^{2} \\
0 \\
0
\end{array}\right] \text {. }
$$

Since the basis functions are piecewise polynomials defined on quasi-uniform regular partition $\tau_{h}$, the coefficient matrix in the system of equation (78) is a large sparse matrix. We use the column principal Gauss elimination method to solve the system. For ease of calculation, we ordered $\alpha=\left(\left(U_{h}\right)^{1},\left(U_{h}\right)^{2}, P_{h}\right)^{T}$. It comes down to just finding a vector $\alpha$ and setting the coefficient matrix to $A 1$ and $B 1$ for the right-hand vector. Since $A 1$ are $2 N G 1+N G 2+1$ rows and $2 N G 1+N G 2$ columns, the column principal Gauss elimination method requires that the coefficient matrix must be a square matrix. There are two ways to convert a matrix into a square matrix. The first is to use the least squares to convert $A 1$ into a square matrix of $2 N G 1+N G 2$; that is, $\left(A 1^{T} \times A 1\right) \alpha=A 1^{T} \times B 1$, and the second is to add the last row of $A 1$ to its last column, set the diagonal element to 1 , and add a variable, i.e.,

$$
\left[\begin{array}{cccc}
A^{11} & A^{12} & A^{13} & 0 \\
A^{21} & A^{22} & A^{23} & 0 \\
A^{31} & A^{32} & A^{33} & A^{43} \\
0 & 0 & A^{43} & 1
\end{array}\right]\left[\begin{array}{c}
\left(U_{h}\right)^{1} \\
\left(U_{h}\right)^{2} \\
P_{h} \\
x
\end{array}\right]=\left[\begin{array}{c}
F^{1} \\
F^{2} \\
0 \\
0
\end{array}\right] .
$$

By transforming $A 1$ into $2 N G 1+N G 2+1$ matrix, we can obtain the solution of the Stokes problem by using the column principal Gauss elimination method.

(1) Types of finite element:

(i) Douglas rotated element shape function: For the velocity $u$, we use the four-node Douglas rotated rectangular element to divide the region $\Omega$ into several combinations of rectangular elements, each element as shown in Figure 1. Let $a_{i}=\left(x_{i}, y_{i}\right), i=1,2,3,4$; then, the centroid coordinates $\left(x_{c}, y_{c}\right)$ are

$x_{c}=\frac{1}{4}\left(x_{1}+x_{2}+x_{3}+x_{4}\right), y_{c}=\frac{1}{4}\left(y_{1}+y_{2}+y_{3}+y_{4}\right)$.

The standard element is a square region of space $(\xi, \eta)$ with sides of 2 : $D:-1 \leq \xi \leq 1$ and $-1 \leq \eta \leq 1$. The centroid coordinates are at the origin $(0,0)$, and its degree of freedom is the value of four points on a square, which are distributed as shown in Figure 1.

Coordinate transformation:

$$
\xi=\frac{\left(x-x_{c}\right)}{a}, \eta=\frac{\left(y-y_{c}\right)}{b} .
$$

Convert the rectangular element $\Omega_{j}$ in the $(x, y)$-plane to the standard element $\widehat{K}$ in the $(\xi, \eta)$-plane, where $2 a=\left|x_{2}-x_{4}\right|$ and $2 b=\left|y_{3}-y_{1}\right|$. Its Jacobi determinant is

$$
|J|=\left|\frac{\partial(x, y)}{\partial(\xi, \eta)}\right|=a b .
$$

Let the shape function of each node of $\widehat{a}_{1}=(0,-1), \widehat{a}_{2}=(1,0), \widehat{a}_{3}=(0,1)$, and $\widehat{a}_{4}=$ $(-1,0)$ be

$$
\begin{aligned}
& \varphi_{1}(\xi, \eta)=\frac{1}{4}-\frac{1}{2} \eta+\frac{3}{8}\left(\left(\xi^{2}-\frac{5}{3} \xi^{4}\right)-\left(\eta^{2}-\frac{5}{3} \eta^{4}\right)\right), \\
& \varphi_{2}(\xi, \eta)=\frac{1}{4}+\frac{1}{2} \xi-\frac{3}{8}\left(\left(\xi^{2}-\frac{5}{3} \xi^{4}\right)-\left(\eta^{2}-\frac{5}{3} \eta^{4}\right)\right), \\
& \varphi_{3}(\xi, \eta)=\frac{1}{4}+\frac{1}{2} \eta+\frac{3}{8}\left(\left(\xi^{2}-\frac{5}{3} \xi^{4}\right)-\left(\eta^{2}-\frac{5}{3} \eta^{4}\right)\right), \\
& \varphi_{4}(\xi, \eta)=\frac{1}{4}-\frac{1}{2} \xi-\frac{3}{8}\left(\left(\xi^{2}-\frac{5}{3} \xi^{4}\right)-\left(\eta^{2}-\frac{5}{3} \eta^{4}\right)\right) .
\end{aligned}
$$

The additional bubble function is $\xi \eta$.

(ii) Piecewise constant shape function: 


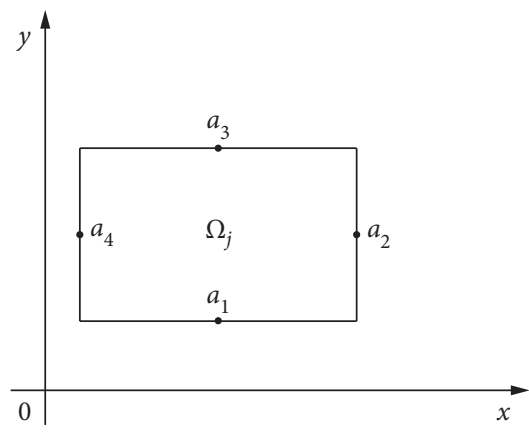

(a)

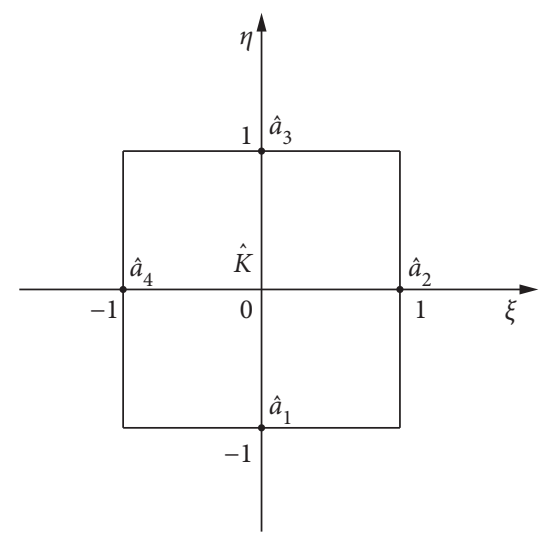

(b)

Figure 1: Transformation from the rectangular element to the standard element for the Douglas rotation rectangular element. (a) Rectangular element $\Omega_{i}$. (b) Standard element $\widehat{K}$.

For the pressure $p$, we use a piecewise constant rectangular element to divide the region $\Omega$ into several combinations of rectangular elements, each of which is shown in Figure 2. Let $a_{1}=$ $\left(x_{c}, y_{c}\right)$ also be the centroid coordinates. The standard rectangular element is a square region in space $(\xi, \eta)$ with a side length of 2 , and let $\widehat{a}_{1}=(0,0)$ also be the centroid coordinates at the origin, as shown in Figure 2.

The coordinate transformation from rectangular element $\Omega_{j}$ to standard element $\widehat{K}$ is the same as for velocity $u$. The node $\widehat{a}_{1}=(0,0)$ corresponds to a shape function

$$
\zeta_{1}(\xi, \eta)=1
$$

(2) Automatic mesh generation of the finite element method:

(i) Automatic mesh generation of the finite element method for velocity $u$ :

In this section, we mainly discuss the automatic generation of 4-node rectangular elements, as well as the node local encoding and the whole encoding comparison table array $I I(4, L E E)$ and the node's actual coordinates' array $X Y(2, N G 1)$. Let the region $\Omega$ be a rectangle, evenly divided by $n$ vertical lines and $m$ horizontal lines; then, the region $\Omega$ is divided into $n \times m$ quadrilateral elements, as shown in Figure 3 .

Figure 3 represents the overall number and the local number of velocity nodes on a small element. The code of the element and the code of the node are arranged from left to right and from bottom to top, so there are $n \times m$ elements and $n(m+1)+(n+1) m$ nodes in the whole area $\Omega$. Use $i, j$ to denote the number of vertical and horizontal lines, respectively. Then, $i$ changes from 0 to $n$, and $j$ changes from 0 to $m$. The coordinates of $(i, j)$ for any node are marked $\left(x_{i}, y_{j}\right)$. Assuming that the middle node of the opposite side of each element is connected by a straight line, there are $2 n+1$ vertical lines and $2 m+1$ horizontal lines in the $\Omega$ region. So, when $i$ changes from 0 to $n$ and $j$ changes from 0 to $2 m$, the coordinates $\left(x_{i}, y_{j}\right)$ can be represented by left-right curved margins XLONG and up-down curved margins YLONG. When $j$ is even,

$$
\left\{\begin{array}{l}
x_{i}=\left(\frac{\mathrm{XLONG}}{n}\right) * \frac{(2 i+1)}{2}, \\
y_{j}=\left(\frac{\mathrm{YLONG}}{m}\right) * \frac{j}{2} .
\end{array}\right.
$$

When $j$ is odd,

$$
\left\{\begin{array}{l}
x_{i}=\left(\frac{\mathrm{XLONG}}{n}\right) * i, \\
y_{j}=\left(\frac{\mathrm{YLONG}}{m}\right) * \frac{j}{2} .
\end{array}\right.
$$

The element whose vertical $i$ element band intersects the horizontal $j$ element band is

$$
L E=j * m+i \text {. }
$$

The number of previous nodes in the $j$ element band is

$N_{j}=j * n+j *(n+1)=(2 n+1) * j$.

Thus, the overall code for the four nodes of element $L E$ is

$$
\begin{array}{llll}
I I(0, L E) & =N_{j}+i ; & I I(1, L E) & =N_{j}+n+i+1 \\
I I(2, L E) & =N_{j}+2 n+1+i ; & I I(3, L E) & =N_{j}+n+i
\end{array} .
$$

(ii) Automatic mesh generation of the finite element method for pressure $p$ : 


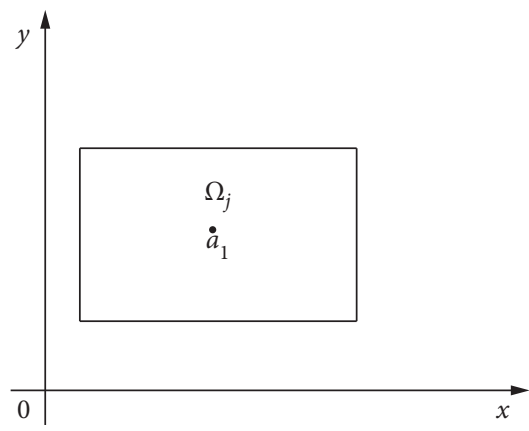

(a)

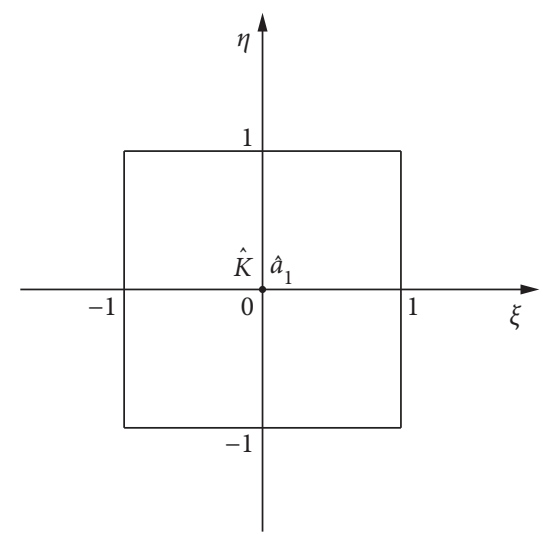

(b)

FiguRe 2: Transformation from the rectangular element to the standard element for the piecewise constant rectangular element. (a) Rectangular element $\Omega_{i}$. (b) Standard element $\widehat{K}$.

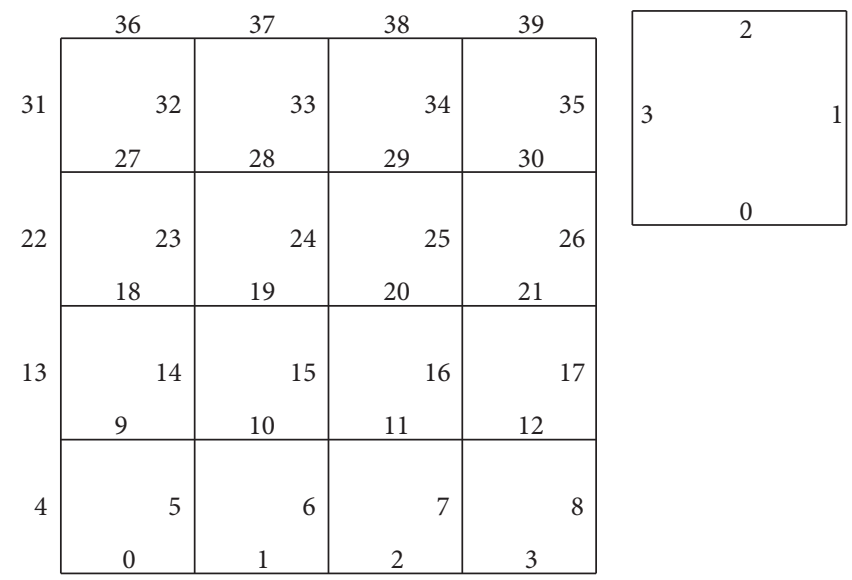

(a)

(b)

Figure 3: Overall number and local number of velocity nodes. (a) Overall number of nodes. (b) Local number of nodes.

In this section, we focus on the automatic generation of 1-node rectangular elements, as well as the node's actual coordinate array $X Y C(2, N G 2)$. If the region $\Omega$ is a rectangle, divided evenly by an $n$ vertical line and an $m$ horizontal line, then the region $\Omega$ is divided into $n \times m$ quadrilateral elements, as shown in Figure 4 .

Figure 4 represents the overall number and the local number of pressure nodes on a small element. The code of the element and the code of the node are arranged from left to right and from bottom to top, so there are $n \times m$ elements and nodes in the whole region $\Omega$. Using $i, j$ to denote the number of vertical and horizontal lines, respectively, then $i$ changes from 0 to $n, j$ changes from 0 to $m$, and each node $(i, j)$ is marked with the coordinates $\left(x_{i}, y_{j}\right)$ which can be represented by the left-right curve space XLONG and the up-down curve space YLONG.

$$
\left\{\begin{array}{l}
x_{i}=\left(\frac{X L O N G}{n}\right) * \frac{(2 i+1)}{2}, \\
y_{j}=\left(\frac{Y L O N G}{m}\right) * \frac{(2 j+1)}{2} .
\end{array}\right.
$$

We have given all the constructions of the matrix in the specific form through the overall coding and local coding and the relationship between the base function and the shape function. We can convert all the integrals into standard units, and then we can do all kinds of integrals on standard units.

We need to deal with the integral. When we do numerical integral, we usually do not use the formula to calculate the integral, but use the higher algebraic precision Gauss-type numerical integral formula. It is not only convenient to write the standard procedure of the integral but also, according to the construction characteristic of the basis function, it is mainly the integral of the polynomial. If the 


\begin{tabular}{|c|c|c|c|}
\hline 12 & 13 & 14 & 15 \\
\hline 8 & 9 & 10 & 11 \\
\hline 4 & 5 & 6 & 7 \\
\hline 0 & 1 & 2 & 3 \\
\hline
\end{tabular}

(a)

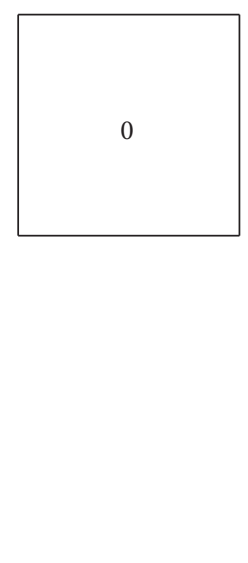

(b)

FIgURE 4: Overall number and local number of pressure nodes. (a) Overall number of nodes. (b) Local number of nodes.

integral is calculated by the formula, it will cause too much rounding error because of the complicated calculation method. Gauss-type integral is to select some special integral point $\xi_{i}$ and weight $H_{i}$ so that the calculation formula

$$
\int_{-1}^{1} f(\xi) \mathrm{d} \xi=\sum_{i=1}^{n} H_{i} f\left(\xi_{i}\right)
$$

is accurate for all $2 n-1$ th polynomials.

We have the same formula for double integrals:

$$
I=\int_{-1}^{1} \int_{-1}^{1} f(\xi, \eta) \mathrm{d} \xi \mathrm{d} \eta
$$

and then $\int_{-1}^{1} f(\xi, \eta) \mathrm{d} \xi=\sum_{j=1}^{n} H_{i} f\left(\xi_{i}, \eta\right)=\phi(\eta)$.

$I=\int_{-1}^{1} \phi(\eta) \mathrm{d} \eta=\sum_{j=1}^{n} H_{j} \phi\left(\eta_{j}\right)=\sum_{j=1}^{n} H_{j}\left(\sum_{i=1}^{n} H_{i} f\left(\xi_{i}, \eta_{j}\right)\right)$.

So,

$$
I=\int_{-1}^{1} \int_{-1}^{1} f(\xi, \eta) \mathrm{d} \xi \mathrm{d} \eta=\sum_{i, j=1}^{n} H_{i} H_{j} f\left(\xi_{i}, \eta_{j}\right) .
$$

With the above analysis, we convert the integrals into double integrals on the standard element and use the 9-node Gauss integral formula; then,

$$
\int_{-1}^{1} \int_{-1}^{1} f(\xi, \eta) \mathrm{d} \xi \mathrm{d} \eta=\sum_{i, j=1}^{n} H_{i} H_{j} f\left(\xi_{i}, \eta_{j}\right)=\sum_{i, j=1}^{n} H_{i j} f\left(\xi_{i}, \eta_{j}\right) .
$$

The coordinates of Gauss point $\xi_{i}, \eta_{j}$ and corresponding weights are

$$
\begin{aligned}
\left(\xi_{i}, \eta_{j}\right) & =(H X(i), H X(j)) \\
1 & \leq i, j \leq 3 H_{i j} \\
& =G H(i) * G H(j) \\
1 & \leq i, j \leq 3
\end{aligned}
$$

where

$$
\begin{aligned}
H X(i) & =(-0.774597,0,0.774597) \\
1 & \leq i, j \leq 3 \\
G H(i) & =(0.555556,0.888889,0.555556) \\
1 & \leq i, j \leq 3 .
\end{aligned}
$$

\section{A Nonconforming Rotated Finite Element Method for the Navier-Stokes Equations}

In the first section of this section, the existence, uniqueness, and stability of the solution of the NRFE approximation problem are discussed. The convergence rate of the NRFE method is given in the second section; there are two steps in solving the Navier-Stokes equations with the NRFE method: dealing with the linear part and dealing with the nonlinear part. The first step has been solved in the last section. Here, we mainly discuss how to solve the nonlinear term by the Newton iteration method. form

Let $(\cdot, \cdot)_{j}=(\cdot, \cdot)_{\Omega_{j}}, 1 \leq j \leq J$, define the discrete bilinear

$$
\begin{aligned}
& a_{h}(u, v)=v \sum_{j=1}^{J}(\nabla u, \nabla v)_{j}, \forall u, v \in X \cup X_{h} \\
& d_{h}(v, q)=\sum_{j=1}^{J}(\nabla \cdot v, q)_{j}, \forall v \in X_{h}, q \in M_{h} .
\end{aligned}
$$


and norm

$$
\|v\|_{1, h}=\left(\sum_{j=1}^{J}\|\nabla v\|_{0, j}^{2}\right)^{1 / 2}=\sqrt{\frac{1}{v} a_{h}(v, v)}, \forall v \in X_{h} .
$$

For $\forall u, v, w \in X \cup X_{h}$, define the discrete trilinear form

$$
\begin{aligned}
b_{1, h}(u, v, w) & =\sum_{j=1}^{J}((u \cdot \nabla) v, w)_{j} \\
b_{h}(u, v, w) & =\frac{1}{2}\left(b_{1, h}(u, v, w)-b_{1, h}(u, w, v)\right),
\end{aligned}
$$

which satisfies the following properties:

(i)

$$
\begin{aligned}
b_{1, h}(u, v, w)+b_{1, h}(u, w, v)= & \sum_{j=1}^{J}\left\langle\left(u \cdot n_{j}\right) v, w\right\rangle_{j} \\
& -\sum_{j=1}^{J}((\nabla \cdot u) v, w)_{j} .
\end{aligned}
$$

(ii)

$$
\begin{aligned}
b_{1, h}(u, v, w)= & b_{h}(u, v, w)+\frac{1}{2} \sum_{j=1}^{J}\left\langle\left(u \cdot n_{j}\right) v, w\right\rangle_{j} \\
& -\frac{1}{2} \sum_{j=1}^{J}((\nabla \cdot u) v, w)_{j} .
\end{aligned}
$$

(iii)

$$
\begin{aligned}
b_{1, h}(u, v, w) & \leq c_{b}\|u\|_{1, h}\|v\|_{1, h}\|w\|_{1, h}, b_{h}(u, v, w) \\
& \leq c_{b}\|u\|_{1, h}\|v\|_{1, h}\|w\|_{1, h} .
\end{aligned}
$$

(iv)

$$
b_{h}(u, v, w)=-b_{h}(u, w, v)
$$

Here and later $n_{j}, 1 \leq j \leq J$, represents the unit outer normal vector of $\partial \Omega_{j}$. See $[7,17]$ for its proofs.

In addition, we introduce a closed subset of $X_{h}$ :

$$
V_{h}=\left\{v \in X_{h} ; d_{h}(v, q)=0, \forall q \in M_{h}\right\} .
$$

Then, the nonconforming rotated finite element approximation problem of problem (6) is

$$
\begin{cases}\text { find }\left(u_{h}, p_{h}\right) \in X_{h} \times M_{h}, & \text { so } \\ a_{h}\left(u_{h}, v\right)-d_{h}\left(v, p_{h}\right)+b_{h}\left(u_{h}, u_{h}, v\right)=(f, v), & \forall v \in X_{h}, \\ d_{h}\left(u_{h}, q\right)=0, & \forall q \in M_{h} .\end{cases}
$$

Theorem 7 (see [7]). Under the condition that Proposition 1 is established, if

$$
\frac{c_{b}}{v^{2}}\|f\|_{*}<1,\|f\|_{*}=\sup _{v \in X_{h}} \frac{(f, v)}{\|v\|_{1, h}},
$$

then there is, to problem (50), a unique solution $\left(u_{h}, p_{h}\right) \in X_{h} \times M_{h}$, and $u_{h} \in V_{h}$ satisfies

$$
\left\|u_{h}\right\|_{1, h} \leq \frac{1}{\nu}\|f\|_{*} .
$$

Proposition 4 (see [7]). Let $(u, p)$ and $\left(u_{h}, p_{h}\right)$ be the solutions of problems (11) and (106), respectively; then, under the condition of formula (51), there exists a positive number $c$ such that

$$
\begin{aligned}
&\left\|u-u_{h}\right\|_{1, h} \leq c\left(\inf _{v \in X_{h}}\|u-v\|_{1, h}+\sup _{v \in V_{h}} \frac{\left|a_{h}(u, v)+b_{h}(u, u, v)-(f, v)\right|}{\|v\|_{1, h}}\right) \\
&\left\|p-p_{h}\right\|_{0} \leq c\left(\inf _{q \in M_{h}}\|p-q\|_{0}+\sup _{v \in X_{h}} \frac{\left|a_{h}(u, v)-d_{h}(v, p)+b_{h}(u, u, v)-(f, v)\right|}{\|v\|_{1, h}}\right. \\
&\left.+\inf _{v \in X_{h}}\|u-v\|_{1, h}+\sup _{v \in V_{h}} \frac{\left|a_{h}(u, v)+b_{h}(u, u, v)-(f, v)\right|}{\|v\|_{1, h}}\right) .
\end{aligned}
$$

Proposition 5 (see [7]). Let $\langle f, g\rangle_{j}=\int_{\partial \Omega_{j}} f g d s$, for $\forall \phi, w \in X \cup X_{h}$, satisfy the following properties: 


$$
\begin{gathered}
\left|\sum_{j=1}^{J}\left\langle\frac{\partial w}{\partial n_{j}}, \phi\right\rangle_{j}\right| \leq c h\|w\|_{2}\|\phi\|_{1, h}, \forall w \in D(A) \\
\left|\sum_{j=1}^{J}\left\langle\left(w \cdot n_{j}\right) v, \phi\right\rangle_{j}\right| \leq c h\|w\|_{1, h}\|v\|_{2}\|\phi\|_{1, h}, \forall v \in H^{2}(\Omega)^{2} \\
\left|\sum_{j=1}^{J}\left\langle q, \phi \cdot n_{j}\right\rangle_{j}\right| \leq c h\|q\|_{1}\|\phi\|_{1, h}, \forall q \in H^{1}(\Omega) .
\end{gathered}
$$$$
\left\|u-u_{h}\right\|_{1, h}+\left\|p-p_{h}\right\|_{0} \leq \operatorname{ch}\left(\left(\|u\|_{2}+\|p\|_{1}\right) .\right.
$$

Using the dual argument method, we estimate the error of $L^{2}$.

Consider the linear duality problem:

$$
\begin{cases}-v \Delta \psi+\nabla \chi+(u \cdot \nabla) \psi+\nabla u \cdot \psi=u-u_{h} & \forall x \in \Omega, \\ \nabla \cdot \psi=0 & \forall x \in \Omega, \\ \psi=0 & \forall x \in \Gamma .\end{cases}
$$

Theorem 8 (see [7]). Let $(u, p) \in H^{2}(\Omega)^{2} \times H^{1}(\Omega)$ and Its variational form is $\left(u_{h}, p_{h}\right) \in X_{h} \times M_{h}$ be the solutions of problems (11) and (106), respectively; then,

$$
\begin{cases}\text { find }(\psi, \chi) \in X \times M, & \text { so } \\ a(v, \psi)-d(v, \chi)+((u \cdot \nabla) v, \psi)+((v \cdot \nabla) u, \psi)=\left(u-u_{h}, v\right), & \forall v \in X \\ d(\psi, q)=0, & \forall q \in M\end{cases}
$$

If $u$ is the nonsingular solution of problem (1), then problem (113) has a unique solution (see [16]).

In order to establish an estimate of the $L^{2}$ error of velocity, the Aubin-Nitsche argumentation method is used here. Let problem (112) be $H^{2}$ regular, i.e.,

$$
\|\psi\|_{2}+\|\chi\|_{1} \leq c\left\|u-u_{h}\right\|_{0}
$$

Let $(\psi, \chi)$ be the solution of problem (113), and $\left(\psi_{h}, \chi_{h}\right) \in X_{h} \times M_{h}$ satisfies

$$
\left\|\psi-\psi_{h}\right\|_{1, h}+\left\|\chi-\chi_{h}\right\|_{0} \leq \operatorname{ch}\left(\|\psi\|_{2}+\|\chi\|_{1}\right) .
$$

Theorem 9 (see [7]). Let $(u, p) \in H^{2}(\Omega)^{2} \times H^{1}(\Omega)$ and $\left(u_{h}, p_{h}\right) \in X_{h} \times M_{h}$ be the solutions of problems (11) and (50), respectively; if problem (112) is $H^{2}$ regular, then

$$
\left\|u-u_{h}\right\|_{0} \leq \operatorname{ch}^{2}\left(\|u\|_{2}+\|p\|_{1}\right)
$$

There are two steps in solving the Navier-Stokes equations using the NRFE method: dealing with the linear part and dealing with the nonlinear part. The method of dealing with the linear term is the same as that in Section 3. When dealing with the nonlinear term, the most common method is the Newton iterative method, and the Newton iterative method requires higher initial value, so we choose the solution of the Stokes problem as the initial value. Let the initial value of the iteration be $\left[u_{h}^{0}, p_{h}^{0}\right]$ and $\left[u_{h}^{n}, p_{h}^{n}\right]$ be the $n$th computing solution; then, the iteration format is as follows:

$$
\left\{\begin{array}{l}
a_{h}\left(u_{h}^{n+1}, v\right)-d_{h}\left(v, p_{h}^{n+1}\right)+b_{h}\left(u_{h}^{n+1}, u_{h}^{n}, v\right)+b_{h}\left(u_{h}^{n}, u_{h}^{n+1}, v\right)=(f, v)+b_{h}\left(u_{h}^{n}, u_{h}^{n}, v\right) \\
\forall v \in X_{h} \\
d_{h}\left(u_{h}^{n+1}, q\right)=0 \\
\forall q \in M_{h} .
\end{array}\right.
$$

In this part, we only need to find the matrix obtained from the nonlinear part and then add it to the matrix obtained from the linear part (i.e., the coefficient matrix of the Stokes equations $A 1$ and the right-hand term $B 1$ ). Then, the solution of the Navier-Stokes equations can be obtained by the iterative method. In the following, we come to explain the concrete process of discretization.

(1) Using $v=\left[\begin{array}{c}\phi_{l} \\ 0\end{array}\right], l=1, \ldots, N G 1$ to discrete equation (117), the iterative partial result is 


$$
\begin{aligned}
b_{h}\left(u_{h}^{n+1}, u_{h}^{n}, v\right) & =\frac{1}{2} \sum_{i=1}^{N G 1} \sum_{j=1}^{N G 1}\left[\left(u_{n+1}^{i}\right)^{1}\left(u_{n}^{j}\right)^{1} \iint_{\Omega}\left(\phi_{i} \frac{\partial \phi_{j}}{\partial x_{1}} \phi_{l}-\phi_{i} \frac{\partial \phi_{l}}{\partial x_{1}} \phi_{j}\right) \mathrm{d} \Omega+\left(u_{n+1}^{i}\right)^{2}\left(u_{n}^{j}\right)^{1} \iint_{\Omega}\left(\phi_{i} \frac{\partial \phi_{j}}{\partial x_{2}} \phi_{l}-\phi_{i} \frac{\partial \phi_{l}}{\partial x_{2}} \phi_{j}\right) \mathrm{d} \Omega\right], \\
b_{h}\left(u_{h}^{n}, u_{h}^{n+1}, v\right) & =\frac{1}{2} \sum_{i=1}^{N G 1} \sum_{j=1}^{N G 1}\left[\left(u_{n}^{j}\right)^{1}\left(u_{n+1}^{i}\right)^{1} \iint_{\Omega}\left(\phi_{j} \frac{\partial \phi_{i}}{\partial x_{1}} \phi_{l}-\phi_{j} \frac{\partial \phi_{l}}{\partial x_{1}} \phi_{i}\right) \mathrm{d} \Omega+\left(u_{n}^{j}\right)^{2}\left(u_{n+1}^{i}\right)^{1} \iint_{\Omega}\left(\phi_{j} \frac{\partial \phi_{i}}{\partial x_{2}} \phi_{l}-\phi_{j} \frac{\partial \phi_{l}}{\partial x_{2}} \phi_{i}\right) \mathrm{d} \Omega\right], \\
b_{h}\left(u_{h}^{n}, u_{h}^{n}, v\right) & =\frac{1}{2} \sum_{i=1}^{N G 1} \sum_{j=1}^{N G 1}\left[\left(u_{n}^{i}\right)^{1}\left(u_{n}^{j}\right)^{1} \iint_{\Omega}\left(\phi_{i} \frac{\partial \phi_{j}}{\partial x_{1}} \phi_{l}-\phi_{i} \frac{\partial \phi_{l}}{\partial x_{1}} \phi_{j}\right) \mathrm{d} \Omega+\left(u_{n}^{i}\right)^{2}\left(u_{n}^{j}\right)^{1} \iint_{\Omega}\left(\phi_{i} \frac{\partial \phi_{j}}{\partial x_{2}} \phi_{l}-\phi_{i} \frac{\partial \phi_{l}}{\partial x_{2}} \phi_{j}\right) \mathrm{d} \Omega\right] .
\end{aligned}
$$

(2) Using $v=\left[\begin{array}{c}0 \\ \phi_{l}\end{array}\right], l=1, \ldots, N G 1$ to discrete equation

(117), the iterative partial result is

$$
\begin{gathered}
b_{h}\left(u_{h}^{n+1}, u_{h}^{n}, v\right)=\frac{1}{2} \sum_{i=1}^{N G 1} \sum_{j=1}^{N G 1}\left[\left(u_{n+1}^{i}\right)^{1}\left(u_{n}^{j}\right)^{2} \iint_{\Omega}\left(\phi_{i} \frac{\partial \phi_{j}}{\partial x_{1}} \phi_{l}-\phi_{i} \frac{\partial \phi_{l}}{\partial x_{1}} \phi_{j}\right) \mathrm{d} \Omega+\left(u_{n+1}^{i}\right)^{2}\left(u_{n}^{j}\right)^{2} \iint_{\Omega}\left(\phi_{i} \frac{\partial \phi_{j}}{\partial x_{2}} \phi_{l}-\phi_{i} \frac{\partial \phi_{l}}{\partial x_{2}} \phi_{j}\right) \mathrm{d} \Omega\right], \\
b_{h}\left(u_{h}^{n}, u_{h}^{n+1}, v\right)=\frac{1}{2} \sum_{i=1}^{N G 1} \sum_{j=1}^{N G 1}\left[\left(u_{n}^{j}\right)^{1}\left(u_{n+1}^{i}\right)^{2} \iint_{\Omega}\left(\phi_{j} \frac{\partial \phi_{i}}{\partial x_{1}} \phi_{l}-\phi_{j} \frac{\partial \phi_{l}}{\partial x_{1}} \phi_{i}\right) \mathrm{d} \Omega+\left(u_{n}^{j}\right)^{2}\left(u_{n+1}^{i}\right)^{2} \iint_{\Omega}\left(\phi_{j} \frac{\partial \phi_{i}}{\partial x_{2}} \phi_{l}-\phi_{j} \frac{\partial \phi_{l}}{\partial x_{2}} \phi_{i}\right) \mathrm{d} \Omega\right], \\
b_{h}\left(u_{h}^{n}, u_{h}^{n}, v\right)=\frac{1}{2} \sum_{i=1}^{N G 1} \sum_{j=1}^{N G 1}\left[\left(u_{n}^{i}\right)^{1}\left(\left(u_{n}^{j}\right)^{2} \iint_{\Omega}\left(\phi_{i} \frac{\partial \phi_{j}}{\partial x_{1}} \phi_{l}-\phi_{i} \frac{\partial \phi_{l}}{\partial x_{1}} \phi_{j}\right) \mathrm{d} \Omega+\left(u_{n}^{i}\right)^{2}\left(u_{n}^{j}\right)^{2} \iint_{\Omega}\left(\phi_{i} \frac{\partial \phi_{j}}{\partial x_{2}} \phi_{l}-\phi_{i} \frac{\partial \phi_{l}}{\partial x_{2}} \phi_{j}\right) \mathrm{d} \Omega\right] .\right.
\end{gathered}
$$

After discretization, the resulting iteration is as follows: where

$$
A 2=\left[\begin{array}{ccc}
A^{11} & A^{12} & 0 \\
A^{21} & A^{22} & 0 \\
0 & 0 & 0 \\
0 & 0 & 0
\end{array}\right] \quad B 2=\left[\begin{array}{c}
F^{1} \\
F^{2} \\
0 \\
0
\end{array}\right],
$$

$$
\begin{aligned}
A^{\tau \omega}= & \left(a_{l i}\right)_{N G 1 \times N G 1}, F^{\tau}=\left(b_{l}\right)_{N G 1} \\
a_{l i}= & \left\{\begin{array}{l}
\frac{1}{2} \sum_{j=1}^{N G 1}\left(u_{n}^{j}\right)^{\sigma} \iint_{\Omega}\left(\phi_{i} \frac{\partial \phi_{j}}{\partial x_{\sigma}} \phi_{l}-\phi_{i} \frac{\partial \phi_{l}}{\partial x_{\sigma}} \phi_{j}\right) \mathrm{d} \Omega+\frac{1}{2} \sum_{j=1}^{N G 1}\left(u_{n}^{j}\right)^{\sigma} \iint_{\Omega}\left(\phi_{j} \frac{\partial \phi_{i}}{\partial x_{\sigma}} \phi_{l}-\phi_{j} \frac{\partial \phi_{l}}{\partial x_{\sigma}} \phi_{i}\right) \mathrm{d} \Omega \tau=\omega \\
\frac{1}{2} \sum_{j=1}^{N G 1}\left(u_{n}^{j}\right)^{\gamma} \iint_{\Omega}\left(\phi_{j} \frac{\partial \phi_{i}}{\partial x_{\gamma}} \phi_{l}-\phi_{j} \frac{\partial \phi_{l}}{\partial x_{\gamma}} \phi_{i}\right) \mathrm{d} \Omega+\frac{1}{2} \sum_{j=1}^{N G 1}\left(u_{n}^{j}\right)^{\tau} \iint_{\Omega}\left(\phi_{i} \frac{\partial \phi_{j}}{\partial x_{\gamma}} \phi_{l}-\phi_{i} \frac{\partial \phi_{l}}{\partial x_{\gamma}} \phi_{j}\right) \mathrm{d} \Omega \tau \neq \omega \\
b_{l}=\frac{1}{2} \sum_{j=1}^{N G 1} \sum_{i=1}^{N G 1}\left[\left(u_{n}^{j}\right)^{\sigma}\left(u_{n}^{i}\right)^{\sigma} \iint_{\Omega}\left(\phi_{j} \frac{\partial \phi_{i}}{\partial x_{\sigma}} \phi_{l}-\phi_{j} \frac{\partial \phi_{l}}{\partial x_{\sigma}} \phi_{i}\right) \mathrm{d} \Omega+\left(u_{n}^{j}\right)^{\gamma}\left(u_{n}^{i}\right)^{\sigma} \iint_{\Omega}\left(\phi_{j} \frac{\partial \phi_{i}}{\partial x_{\gamma}} \phi_{l}-\phi_{j} \frac{\partial \phi_{l}}{\partial x_{\gamma}} \phi_{i}\right) \mathrm{d} \Omega\right] .
\end{array}\right.
\end{aligned}
$$

In the formula above, $\tau, \omega, \sigma, \gamma=1,2$, and $\sigma \neq \gamma$. 
In this way, we can construct a finite element system of equations, $(A 1+A 2) \alpha^{m+1}=B 1+B 2$, and solve it iteratively. Until $\left\|\alpha^{m+1}-\alpha^{m}\right\|$ is less than given $\varepsilon$, we can get the solution $u_{h}, p_{h}$ of equation (64).

\section{A Two-Level Nonconforming Rotated Finite Element Method for the Navier-Stokes Equations}

Because of the nonlinear and incompressible conditions of the Navier-Stokes equations, it is very difficult to solve them numerically, and they consume a lot of CPU time. To overcome these difficulties, many methods have been proposed, including the two-level method. The aim of the twolevel method is to obtain the approximate solution of the nonlinear equation in less time and to maintain the optimal convergence speed. The concrete calculation is to solve a nonlinear problem on the coarse grid and a linear problem on the fine grid. The theoretical and numerical experiments in this paper show that, compared with the NRFE method, the TNRFE method can save a lot of time when the convergence rate reaches the same order, which shows that it is an effective algorithm. In this section, we prove the stability and convergence rate of the TNRFE method.

We choose a coarse grid $H$ and a fine grid $h$ with $H \gg 0, H, h$ to represent the coarse and fine grid scales of the velocity and pressure spaces and then establish a nonconforming finite element space $X_{H} \times M_{H}, X_{h} \times M_{h}$, and $X_{H} \times M_{H} \subset X_{h} \times M_{h}$. Similarly, they satisfy all the properties mentioned in the previous section.

The Newton two-level nonconforming finite element method is divided into two steps:

Step 1: solving a nonlinear Navier-Stokes problem on a coarse grid:

$$
\begin{cases}\text { find }\left(u_{H}, p_{H}\right) \in X_{H} \times M_{H}, & \text { so } \\ a_{h}\left(u_{H}, v\right)-d_{h}\left(v, p_{H}\right)+d_{h}\left(u_{H}, q\right)+b_{h}\left(u_{H}, u_{H}, v\right)=(f, v), & \forall(v, q) \in X_{H} \times M_{H} .\end{cases}
$$

Step 2: solving a linearized Navier-Stokes problem on a fine grid:

$$
\begin{cases}\operatorname{find}\left(u^{h}, p^{h}\right) \in X_{h} \times M_{h} & \text { so } \\ a_{h}\left(u^{h}, v\right)-d_{h}\left(v, p^{h}\right)+d_{h}\left(u^{h}, q\right)+b_{h}\left(u^{h}, u_{H}, v\right)+b_{h}\left(u_{H}, u^{h}, v\right)=(f, v)+b_{h}\left(u_{H}, u_{H}, v\right), & \forall(v, q) \in X_{h} \times M_{h} .\end{cases}
$$

Define a generalized bilinear form on $(X \times M) \times(X \times M):$

$$
\mathscr{B}((u, p) ;(v, q))=a(u, v)-d(v, p)+d(u, q) .
$$

So, the variational form of problem (1) is as follows: find $(u, p) \in X \times M$ such that

$$
\mathscr{B}((u, p) ;(v, q))+b(u, u, v)=(f, v), \forall(v, q) \in X \times M .
$$

For given $(u, p) \in X \times M$, we define its Stokes projection $(w, \pi) \in X_{h} \times M_{h}$ as

$$
\mathscr{B}_{h}((w, \pi) ;(v, q))=\mathscr{B}((u, p) ;(v, q)), \forall(v, q) \in X_{h} \times M_{h} .
$$

Then, nonconforming rotated finite element approximation problem (124) is as follows: find $\left(u_{h}, p_{h}\right) \in X_{h} \times M_{h}$ so that

$$
\mathscr{B}_{h}\left(\left(u_{h}, p_{h}\right) ;(v, q)\right)+b_{h}\left(u_{h}, u_{h}, v\right)=(f, v), \forall(v, q) \in X_{h} \times M_{h} .
$$

Proposition 6. (i) There is a constant $c>0$ such that

$$
|\mathscr{B}((u, p) ;(v, q))| \leq c\left(\|\nabla u\|_{0}+\|p\|_{0}\right)\left(\|\nabla v\|_{0}+\|q\|_{0}\right), \forall(u, p),(v, q) \in X \times M
$$

(ii) There exists a constant $\alpha>0$ that depends on $h$ :

$$
\alpha\left(\left\|u_{h}\right\|_{1, h}+\left\|p_{h}\right\|_{0}\right) \leq \sup \left(v_{h}, q_{h}\right) \in X_{h} \times M_{h} \frac{\mathscr{B}_{h}\left(\left(u_{h}, p_{h}\right) ;\left(v_{h}, q_{h}\right)\right)}{\left\|v_{h}\right\|_{1, h}+\left\|q_{h}\right\|_{0}}, \forall\left(u_{h}, p_{h}\right) \in X_{h} \times M_{h} .
$$


Proof. ((70) proof (see [4, 16-18])). The following is to prove (129). Let $\left(u_{h}, p_{h}\right)$ be a nonzero element in $X_{h} \times M_{h}$.
Let $q_{h} \in M_{h}$ be determined by the problem $d_{h}\left(\psi_{h}, p_{h}\right)=d_{h}\left(\psi_{h}, q_{h}\right), \forall \psi_{h} \in X_{h}$, so

$$
\mathscr{B}_{h}\left(\left(u_{h}, p_{h}\right) ;\left(u_{h}, q_{h}\right)\right)=v\left\|u_{h}\right\|_{1, h}^{2}-d_{h}\left(u_{h}, p_{h}\right)+d_{h}\left(u_{h}, q_{h}\right)=v\left\|u_{h}\right\|_{1, h}^{2}
$$

Since there exists the constant $\delta>0$ for all $p_{h} \in M_{h} \subset M$, there exists $v \in X$ satisfying $\nabla \cdot v=-p_{h}$ and $\delta\|\nabla v\|_{0} \leq\left\|p_{h}\right\|_{0}$.
Hence, derived from upper equations (39) and (40) and the Young inequality,

$$
\begin{aligned}
\mathscr{B}_{h}\left(\left(u_{h}, p_{h}\right) ;\left(\Pi_{h} v, 0\right)\right) & \geq-v\left\|u_{h}\right\|_{1, h}\left\|\Pi_{h} v\right\|_{1, h}-d_{h}\left(\Pi_{h} v, p_{h}\right) \\
& \geq-c v\left\|u_{h}\right\|_{1, h}\|\nabla v\|_{0}-d_{h}\left(\Pi_{h} v-v, p_{h}\right)+\left\|p_{h}\right\|_{0}^{2} \\
& \geq-c v\left\|u_{h}\right\|_{1, h}\|\nabla v\|_{0}+\left\|p_{h}\right\|_{0}^{2} \\
& \geq-\frac{c^{2} v^{2}}{2 \delta}\left\|u_{h}\right\|_{1, h}^{2}-\frac{\delta}{2}\|\nabla v\|_{0}^{2}+\left\|p_{h}\right\|_{0}^{2} \\
& \geq-\frac{c^{2} v^{2}}{2 \delta}\left\|u_{h}\right\|_{1, h}^{2}+\frac{1}{2}\left\|p_{h}\right\|_{0}^{2} .
\end{aligned}
$$

Let $w_{h}=u_{h}+\left(\delta / c^{2} v\right) \Pi_{h} v$ and $r_{h}=q_{h}$; from the above two formulas, we can get

$$
\mathscr{B}_{h}\left(\left(u_{h}, p_{h}\right) ;\left(w_{h}, r_{h}\right)\right) \geq \frac{v}{2}\left\|u_{h}\right\|_{1, h}^{2}+\frac{\delta}{2 c^{2} v}\left\|p_{h}\right\|_{0}^{2} .
$$

Obtained from (50) and (40), the triangle inequality, and the Hölder inequality,

$$
\begin{aligned}
& \left\|w_{h}\right\|_{1, h}+\left\|r_{h}\right\|_{0} \\
& =\left\|u_{h}+\frac{\delta}{c^{2} v} \Pi_{h} v\right\|_{1, h}+\left\|q_{h}\right\|_{0} \\
& \leq\left\|u_{h}\right\|_{1, h}+\frac{\delta}{c^{2} v}\left\|\Pi_{h} v\right\|_{1, h}+\frac{1}{\beta_{0}} \sup _{\psi_{h} \in X_{h}} \frac{d_{h}\left(\psi_{h}, q_{h}\right)}{\left\|\psi_{h}\right\|_{1, h}} \\
& \leq\left\|u_{h}\right\|_{1, h}+\frac{\delta}{c \nu}\|\nabla v\|_{0}+\frac{1}{\beta_{0}} \sup _{\psi_{h} \in X_{h}} \frac{d_{h}\left(\psi_{h}, p_{h}\right)}{\left\|\psi_{h}\right\|_{1, h}} \\
& \leq\left\|u_{h}\right\|_{1, h}+\frac{1}{c \nu}\left\|p_{h}\right\|_{0}+\frac{\sqrt{2}}{\beta_{0}}\left\|p_{h}\right\|_{0} .
\end{aligned}
$$

From the above two formulas, it is easy to get (129).

Theorem 10. Under the conditions of Proposition 6 and Theorem 7 , the solution $\left(u^{h}, p^{h}\right) \in X_{h} \times M_{h}$ satisfies

$$
\left\|u^{h}\right\|_{1, h}+\left\|p^{h}\right\|_{0} \leq c\|f\|_{*} .
$$

Here, $c$ is a constant that depends on $\nu, f$, and $\Omega$.
Proof. According to Theorem 7 and (122), there exists a unique solution $\left(u_{H}, p_{H}\right) \in X_{H} \times M_{H}$, and it satisfies

$$
\left\|u_{H}\right\|_{1, h} \leq \frac{1}{\nu}\|f\|_{*} .
$$

In equation (123), we make $v=u^{h}$ and $q=p^{h}$; then, from (104), we can derive

$v\left\|u^{h}\right\|_{1, h}^{2}+b_{h}\left(u^{h}, u_{H}, u^{h}\right)=\left(f, u^{h}\right)+b_{h}\left(u_{H}, u_{H}, u^{h}\right)$.

Using (103), we can get

$v\left\|u^{h}\right\|_{1, h}^{2} \leq c_{b}\left\|u^{h}\right\|_{1, h}^{2}\left\|u_{H}\right\|_{1, h}+\|f\|_{*}\left\|u^{h}\right\|_{1, h}+c_{b}\left\|u_{H}\right\|_{1, h}^{2}\left\|u^{h}\right\|_{1, h}$.

From (135), we can derive

$v\left\|u^{h}\right\|_{1, h}^{2} \leq \frac{c_{b}}{v}\|f\|_{*}\left\|u^{h}\right\|_{1, h}^{2}+\|f\|_{*}\left\|u^{h}\right\|_{1, h}+\frac{c_{b}}{v^{2}}\|f\|_{*}^{2}\left\|u^{h}\right\|_{1, h}$.

After sorting through the equations, we can get

$$
\nu\left(1-\frac{c_{b}}{v^{2}}\|f\|_{*}\right)\left\|u^{h}\right\|_{1, h} \leq\left(1+\frac{c_{b}}{v^{2}}\|f\|_{*}\right)\|f\|_{*} .
$$

From Theorem 7, we can get

$$
\left\|u^{h}\right\|_{1, h} \leq c\|f\|_{*} .
$$

From Proposition 6 and (123), we can get 


$$
\begin{aligned}
& \alpha\left(\left\|u^{h}\right\|_{1, h}+\left\|p^{h}\right\|_{0}\right) \\
& \quad \leq \sup _{(v, q) \in X_{h} \times M_{h}} \frac{\mathscr{B}_{h}\left(\left(u^{h}, p^{h}\right) ;(v, q)\right)}{\|v\|_{1, h}+\|q\|_{0}} \\
& \quad \leq c_{b}\left\|u_{H}\right\|_{1, h}^{2}+\|f\|_{*}+2 c_{b}\left\|u^{h}\right\|_{1, h}\left\|u_{H}\right\|_{1, h} .
\end{aligned}
$$

From (135), (140), and (141), we can derive

$$
\left\|u^{h}\right\|_{1, h}+\left\|p^{h}\right\|_{0} \leq c\|f\|_{*} .
$$

Theorem 11. Under the conditions of Proposition 6 and Theorem 7 , there exists a constant $c$ which has nothing to do with $h$ such that the solution $\left(u^{h}, p^{h}\right)$ of problems (122) and (123) satisfies

$$
\left\|u-u^{h}\right\|_{1, h}+\left\|p-p^{h}\right\|_{0} \leq c\left(h+H^{2}\right) .
$$

Proof. Multiply the two ends of the first formula of (1) by $v \in X_{h}$ and (102), and we can get

$$
a_{h}(u, v)-d_{h}(v, p)+b_{h}(u, u, v)-v \sum_{j=1}^{J}\left\langle\frac{\partial u}{\partial n_{j}}, v\right\rangle_{j}+\sum_{j=1}^{J}\left\langle p, v \cdot n_{j}\right\rangle_{j}+\frac{1}{2} \sum_{j=1}^{J}\left\langle\left(u \cdot n_{j}\right) u, v\right\rangle_{j}=(f, v) .
$$

Suppose $(w, \pi)$ is the projection of $(u, p)$ on $X_{h} \times M_{h}$, and here, $w \in V_{h}$; then, by (125) and (144), we can get

$$
\begin{aligned}
& a_{h}\left(u^{h}-w, v\right)-d_{h}\left(v, p^{h}-\pi\right)+d_{h}\left(u^{h}-w, q\right)=\mathscr{B}_{h}\left(\left(u^{h}-w, p^{h}-\pi\right) ;(v, q)\right) \\
& =b_{h}(u, u, v)-v \sum_{j=1}^{J}\left\langle\frac{\partial u}{\partial n_{j}}, v\right\rangle_{j}+\sum_{j=1}^{J}\left\langle p, v \cdot n_{j}\right\rangle_{j}+\frac{1}{2} \sum_{j=1}^{J}\left\langle\left(u \cdot n_{j}\right) u, v\right\rangle_{j} \\
& \quad+b_{h}\left(u_{H}, u_{H}, v\right)-b_{h}\left(u^{h}, u_{H}, v\right)-b_{h}\left(u_{H}, u^{h}, v\right) .
\end{aligned}
$$

In (145), we take $v=u^{h}-w$ and $q=p^{h}-\pi$. By using (3),

(103), and Proposition 5, we can get

$$
\begin{aligned}
v\left\|u^{h}-w\right\|_{1, h}^{2}= & b_{h}\left(u, u, u^{h}-w\right) \\
& -v \sum_{j=1}^{J}\left\langle\frac{\partial u}{\partial n_{j}}, u^{h}-w\right\rangle_{j}+\sum_{j=1}^{J}\left\langle p,\left(u^{h}-w\right) \cdot n_{j}\right\rangle_{j} \\
& +\frac{1}{2} \sum_{j=1}^{J}\left\langle\left(u \cdot n_{j}\right) u, u^{h}-w\right\rangle_{j} \\
& +b_{h}\left(u_{H}, u_{H}, u^{h}-w\right)-b_{h}\left(u^{h}, u_{H}, u^{h}-w\right)-b_{h}\left(u_{H}, u^{h}, u^{h}-w\right) \\
= & b_{h}\left(u-w, u, u^{h}-w\right)+b_{h}\left(w, u-w, u^{h}-w\right)-b_{h}\left(u^{h}-w, u_{H}, u^{h}-w\right) \\
& -b_{h}\left(u_{H}-w, u^{h}-w, u_{H}-w\right)-v \sum_{j=1}^{J}\left\langle\frac{\partial u}{\partial n_{j}}, u^{h}-w\right\rangle_{j} \\
& +\sum_{j=1}^{J}\left\langle p,\left(u^{h}-w\right) \cdot n_{j}\right\rangle_{j}+\frac{1}{2} \sum_{j=1}^{J}\left\langle\left(u \cdot n_{j}\right) u, u^{h}-w\right\rangle_{j} \\
\leq & c_{b}\left\|u^{h}-w\right\|_{1, h}^{2}\left\|u_{H}\right\|_{1, h}+c_{b}\left\|u^{h}-w\right\|_{1, h}\left(\|u-w\|_{1, h}\|\nabla u\|_{0}+\|w\|_{1, h}\|u-w\|_{1, h}+\left\|u_{H}-w\right\|_{1, h}^{2}\right) \\
& +c h\left\|u^{h}-w\right\|_{1, h}\left(\|u\|_{2}+\|p\|_{1}+\|\nabla u\|_{0}\|u\|_{2}\right) .
\end{aligned}
$$


From Theorems 2, 3, 7, and 8 and (135), we can get

$$
\left\|u^{h}-w\right\|_{1, h} \leq c\left(h+H^{2}\right) .
$$

$$
\begin{aligned}
\| u^{h}- & w\left\|_{1, h}+\right\| p^{h}-\pi \|_{0} \leq \alpha^{-1} \sup _{(v, q) \in X_{h} \times M_{h}} \frac{\mathscr{B}_{h}\left(\left(u^{h}-w, p^{h}-\pi\right) ;(v, q)\right)}{\|v\|_{1, h}+\|q\|_{0}} \\
\leq & c\left(\|\nabla u\|_{0}+\left\|u^{h}-w\right\|_{1, h}+\|w\|_{1, h}\right)\left(\|u-w\|_{1, h}+\left\|u^{h}-w\right\|_{1, h}\right) \\
& +c\left(\left\|u^{h}-w\right\|_{1, h}+\left\|w-u_{H}\right\|_{1, h}\right)\left\|u^{h}-w\right\|_{1, h}+c\left\|w-u_{H}\right\|_{1, h}^{2} \\
& +c h\left(\|u\|_{2}+\|p\|_{1}+\|\nabla u\|_{0}\|u\|_{2}\right) \leq c\left(h+H^{2}\right) .
\end{aligned}
$$

So,

$$
\begin{aligned}
\| u & -u^{h}\left\|_{1, h}+\right\| p-p^{h} \|_{0} \\
& \leq\|u-w\|_{1, h}+\left\|w-u^{h}\right\|_{1, h}+\|p-\pi\|_{0}+\left\|\pi-p^{h}\right\|_{0} \\
& \leq c\left(h+H^{2}\right) .
\end{aligned}
$$

From the above results, when the appropriate scaling relation is chosen, i.e., $h=O\left(H^{2}\right)$, we can see that the NRFE method and the Newton TNRFE method have the same convergence rate.

\section{Computational Example}

Here, examples are given to verify the correctness of the theory and to show that the two-level nonconforming finite element method for solving the Navier-Stokes equations is superior to the nonconforming finite element method.

Example 1. Let us take a true solution which satisfies the conditions and set the two-dimensional rectangular region $\Omega=[0,1] \times[0,1]$; the true solutions are as follows:

$$
\left\{\begin{array}{l}
u(x, y)=\left(u_{1}(x, y), u_{2}(x, y)\right), \\
u_{1}(x, y)=x^{2}(x-1)^{2} y(y-1)(2 y-1), \\
u_{2}(x, y)=-x(x-1)(2 x-1) y^{2}(y-1)^{2}, \\
p(x, y)=x-0.5 .
\end{array}\right.
$$

It is proved that the velocity term of this vector function belongs to the nondivergence space, i.e., $\nabla \cdot u=0$, and the pressure term satisfies $\int_{\Omega} p(x) \mathrm{d} x=0$. By substituting $u(x, y), p(x, y)$ into Stokes (12), the right-hand term $f(x, y)=\left(f_{1}(x, y), f_{2}(x, y)\right)$ is obtained. Since the viscosity coefficient has little effect on the accuracy of the equation, we only consider the case when the viscosity coefficient is $v=1$. The numerical results of the nonconforming finite element method with different values of $h$ are given in Table 1.

From Table 1, for solving the Stokes equations, we can see that the NRFE method converges with $h$ decreasing, and it keeps the convergence rate. When the grid scale is $h=1 / 25$, the true solution and the calculation solution of the velocity component $u_{1}$ are shown in Figure 5.
The contour of the true solution and the calculation solution of the velocity component $u_{1}$ is shown in Figure 6 .

The true solution and the calculation solution of the velocity component $u_{2}$ are shown in Figure 7 .

The contour of the true solution and the calculation solution of the velocity component $u_{2}$ is shown in Figure 8 .

The vector of the true solution and the calculation solution of the velocity is shown in Figure 9.

The images of the true solution and the calculation solution of the pressure are shown in Figure 10.

The contours of the true solution and the calculated solution of the pressure are shown in Figure 11.

Here, examples are given to verify the correctness of the theory and to show that the TNRFE method for solving the Navier-Stokes equations is superior to the NRFE method.

Example 2. Let us take a true solution which satisfies the conditions, and let the two-dimensional rectangular region $\Omega=[0,1] \times\lfloor 0,1\rfloor$; the true solution is as follows:

$$
\left\{\begin{array}{l}
u(x, y)=\left(u_{1}(x, y), u_{2}(x, y)\right), \\
u_{1}(x, y)=10 x^{2}(x-1)^{2} y(y-1)(2 y-1), \\
u_{2}(x, y)=-10 x(x-1)(2 x-1) y^{2}(y-1)^{2}, \\
p(x, y)=10(2 x-1)(2 y-1) .
\end{array}\right.
$$

It is proved that the velocity term of this vector function belongs to the nondivergence space, i.e., $\nabla \cdot u=0$, and the pressure term satisfies $\int_{\Omega} p(x) \mathrm{d} x=0$. By substituting $u(x, y), p(x, y)$ into Navier-Stokes equation (1), the righthand term of the equation can be obtained: $f(x, y)=\left(f_{1}(x, y), f_{2}(x, y)\right)$. Since the viscosity coefficient has little effect on the accuracy of the equation, we only consider the case when the viscosity coefficient is $v=1$. In this paper, we present the numerical results of the TNRFE method and the NRFE method when the value of $h$ is different.

When the grid scale is $h=1 / 25$, the true solution and the calculation solution of the velocity component $u_{1}$ are shown in Figure 12.

The contour of the true solution and the calculation solution of the velocity component $u_{1}$ is shown in Figure 13 .

The true solution and the calculation solution of the velocity component $u_{2}$ are shown in Figure 14 . 
TABLE 1: Convergence study of the NRFE method for the Stokes equations in Example 1.

\begin{tabular}{lcccc}
\hline$h$ & CPU $(\mathrm{s})$ & $\left\|u-u_{h}\right\|_{1, h} /\|u\|_{1, h}$ & $\left\|u-u_{h}\right\|_{0} /\|u\|_{0}$ & $\left\|p-p_{h}\right\|_{0} /\|p\|_{0}$ \\
\hline $1 / 9$ & 1 & 0.05276735294 & 0.00683378123 & 0.00721657884 \\
$1 / 16$ & 14 & 0.04930544340 & 0.00595934426 & 0.00672908995 \\
$1 / 25$ & 249 & 0.04626780379 & 0.00524238897 & 0.00630336367 \\
\hline
\end{tabular}

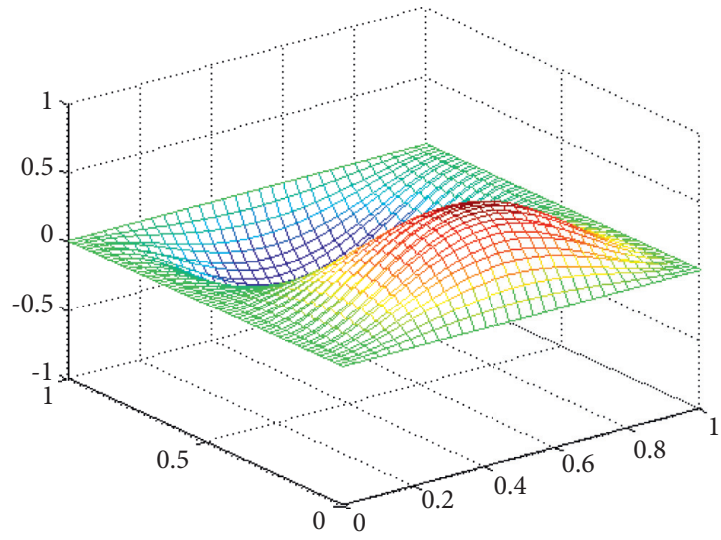

(a)

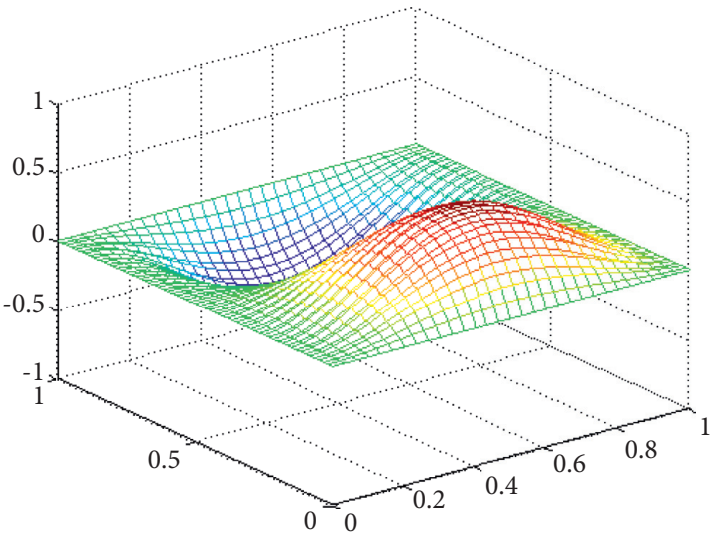

(b)

FIGURE 5: True solution and calculation solution of velocity component $u_{1}$ by the NRFE method for the Stokes equations. (a) True solution of velocity component $u_{1}$. (b) Calculation solution of velocity component $u_{1}$.

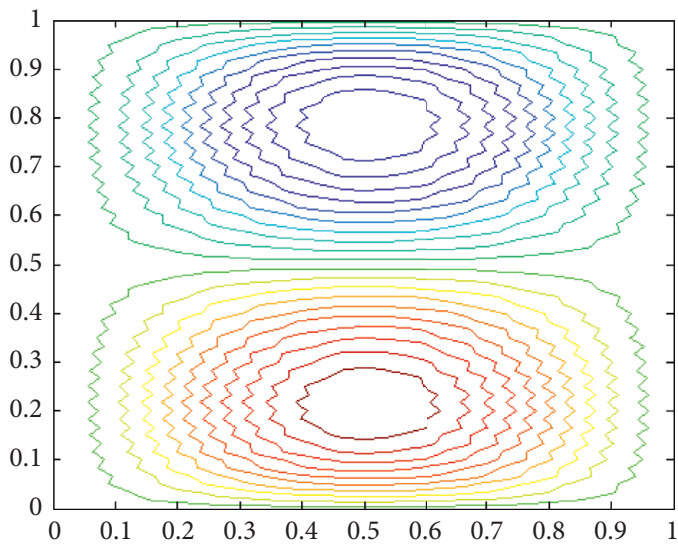

(a)

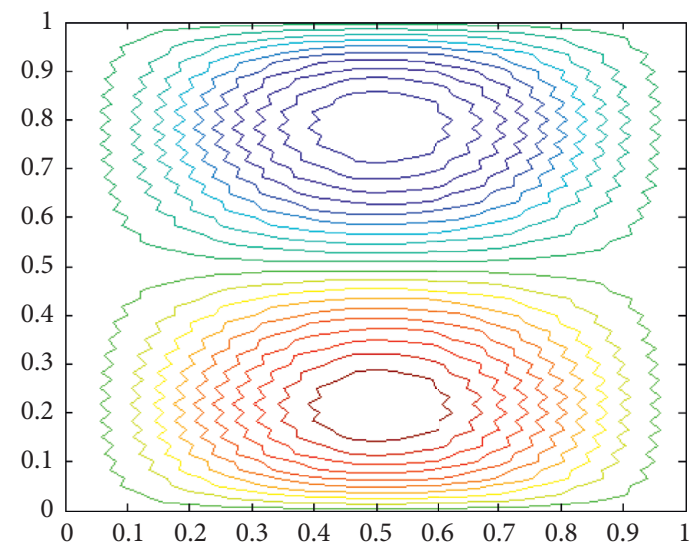

(b)

Figure 6: True solution contour and calculation solution contour of velocity component $u_{1}$ by the NRFE method for the Stokes equations. (a) True solution contour of velocity component $u_{1}$. (b) Calculation solution contour of velocity component $u_{1}$.

The contour of the true solution and the calculation solution of the velocity component $u_{2}$ is shown in Figure 15.

The vector of the true solution and the calculation solution of the velocity is shown in Figure 16.

The images of the true solution and the calculation solution of the pressure are shown in Figure 17.

The contours of the true solution and the calculation solution of the pressure are shown in Figure 18.

From Tables 2 and 3, we can see that the NRFE method and the TNRFE method have the same order convergence speed when $h=O\left(H^{2}\right)$ for solving the Navier-Stokes equations. The TNRFE method we proposed is less timeconsuming than the NRFE method; actually, it nearly saves half the CPU time. We plot the velocity by tracing diagonal points. When the grid scale is $H=1 / 5$ and $h=1 / 25$, the true solution and the computational solution of velocity component $u_{1}$ are represented in Figure 19.

The contour of the true solution and the calculation solution of the velocity component $u_{1}$ is shown in Figure 20 .

The true solution and the calculation solution of the velocity component $u_{2}$ are shown in Figure 21 .

The contour of the true solution and the calculated solution of the velocity component $u_{2}$ is shown in Figure 22 .

The vector of the true solution and the calculated solution of the velocity is shown in Figure 23.

The images of the true solution and the calculated solution of the pressure are shown in Figure 24. 


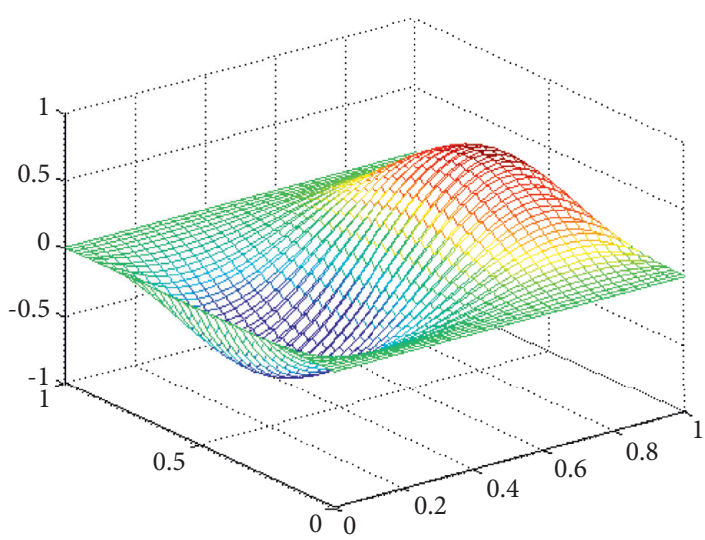

(a)

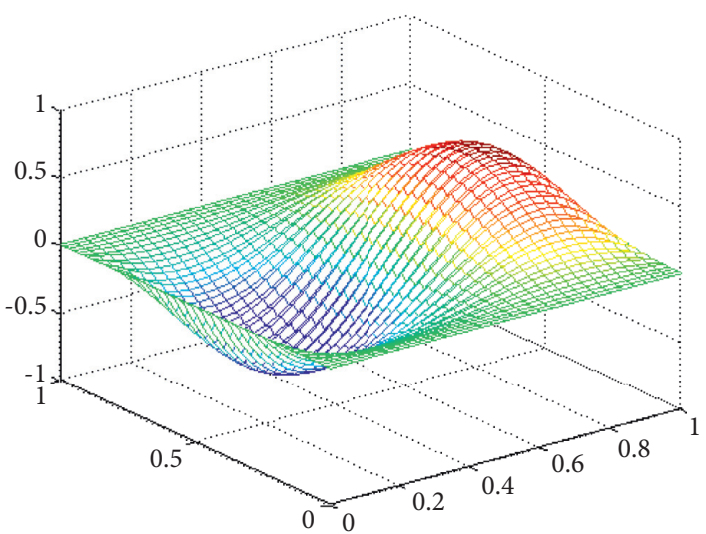

(b)

FIgURE 7: True solution and calculation solution of velocity component $u_{2}$ by the NRFE method for the Stokes equations. (a) True solution of velocity component $u_{2}$. (b) Calculation solution of velocity component $u_{2}$.

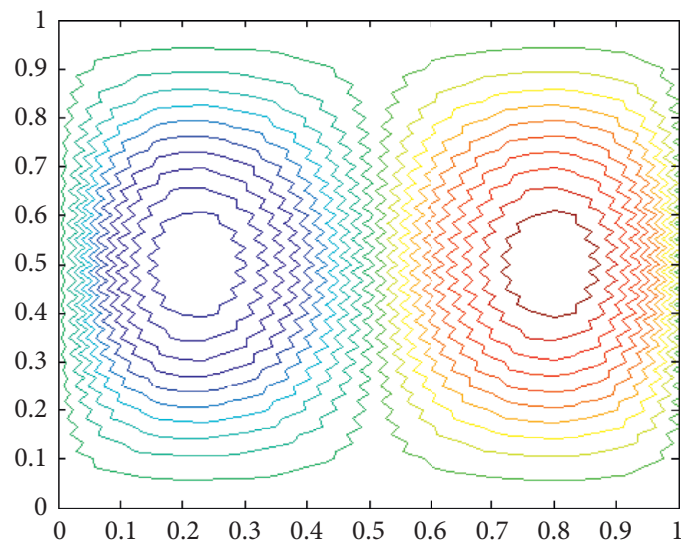

(a)

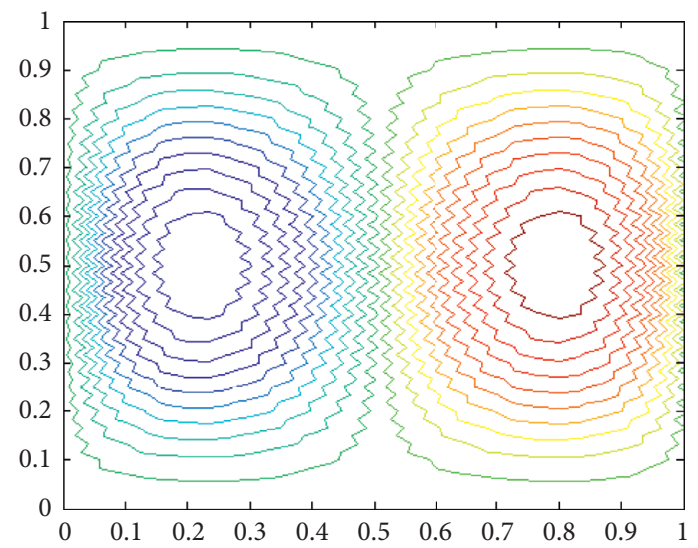

(b)

FIGURE 8: True solution contour and calculation solution contour of velocity component $u_{2}$ by the NRFE method for the Stokes equations. (a) True solution contour of velocity component $u_{2}$. (b) Calculation solution contour of velocity component $u_{2}$.

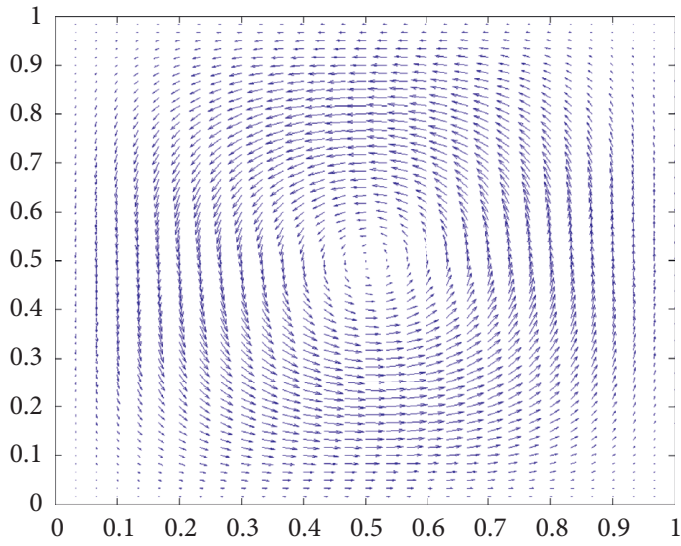

(a)

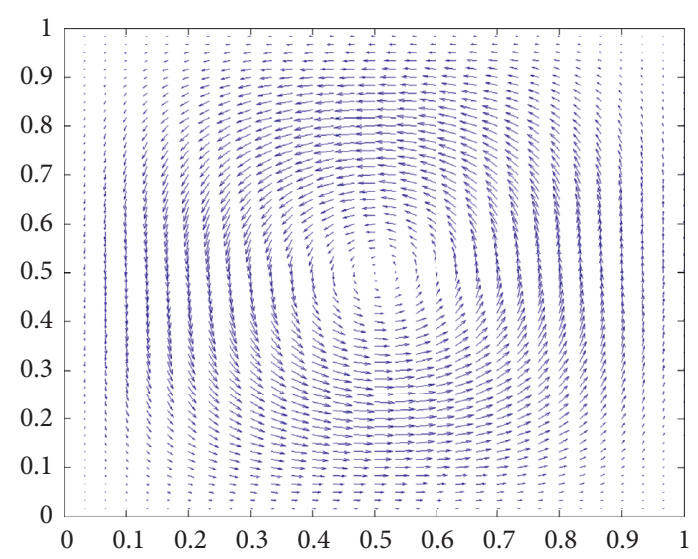

(b)

FIgURE 9: True solution vector and calculation solution vector of velocity by the NRFE method for the Stokes equations. (a) True solution vector of velocity. (b) Calculation solution vector of velocity. 


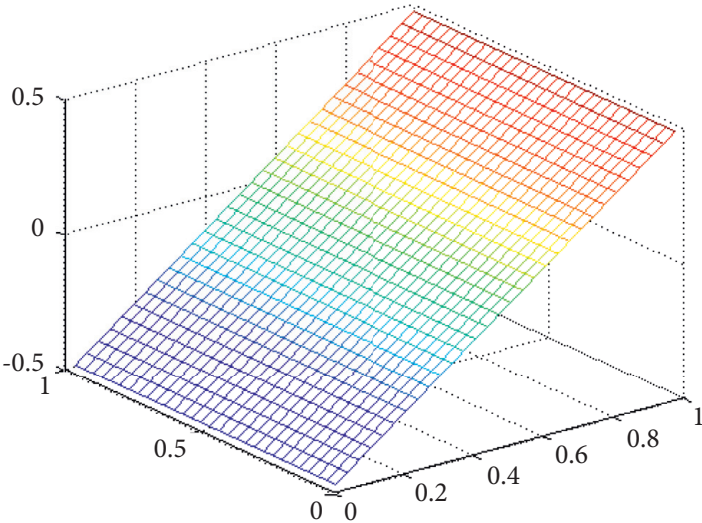

(a)

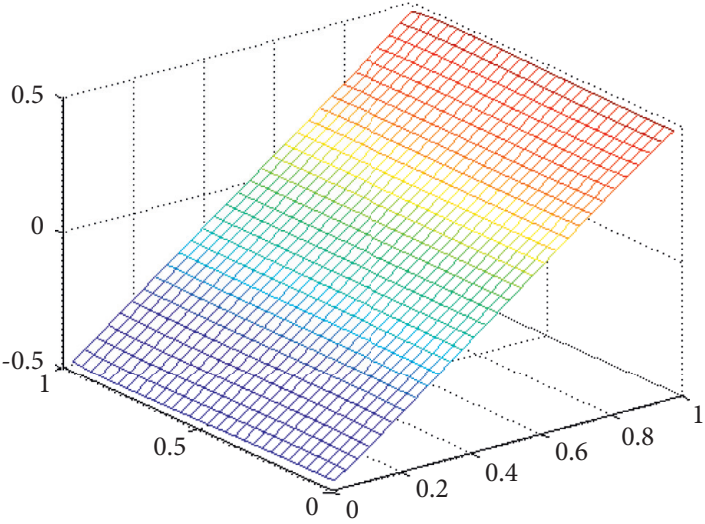

(b)

Figure 10: True solution and calculation solution of pressure by the NRFE method for the Stokes equations. (a) True solution of pressure. (b) Calculation solution of pressure.

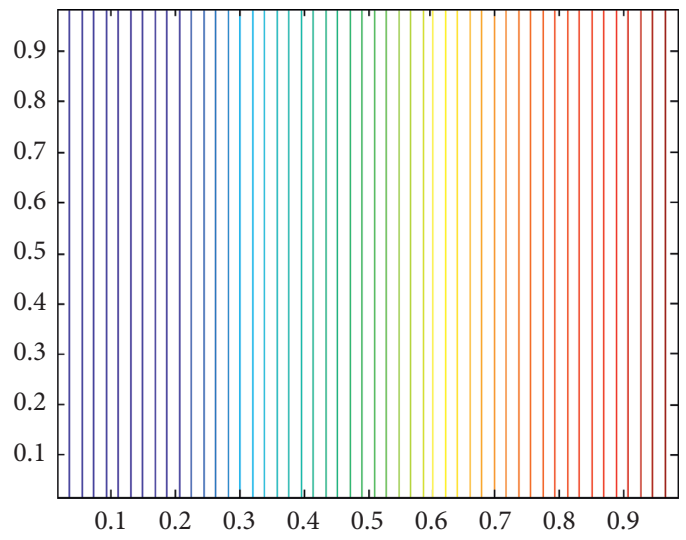

(a)

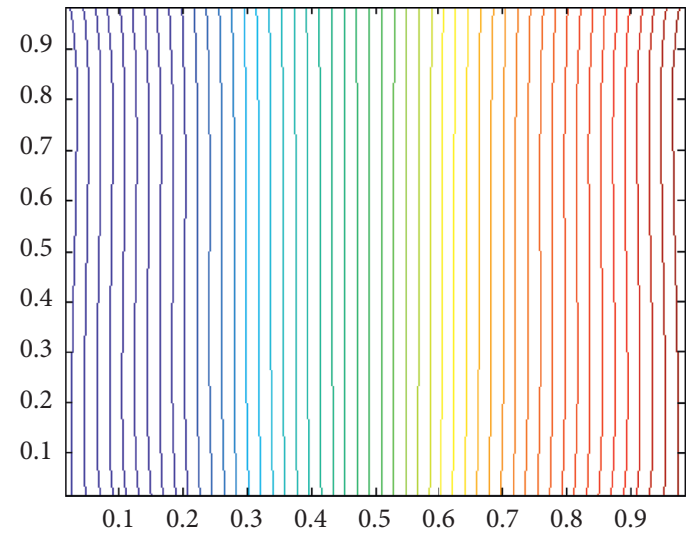

(b)

FIGURE 11: True solution contour and calculation solution contour of pressure by the NRFE method for the Stokes equations. (a) True solution contour of pressure. (b) Calculation solution contour of pressure.

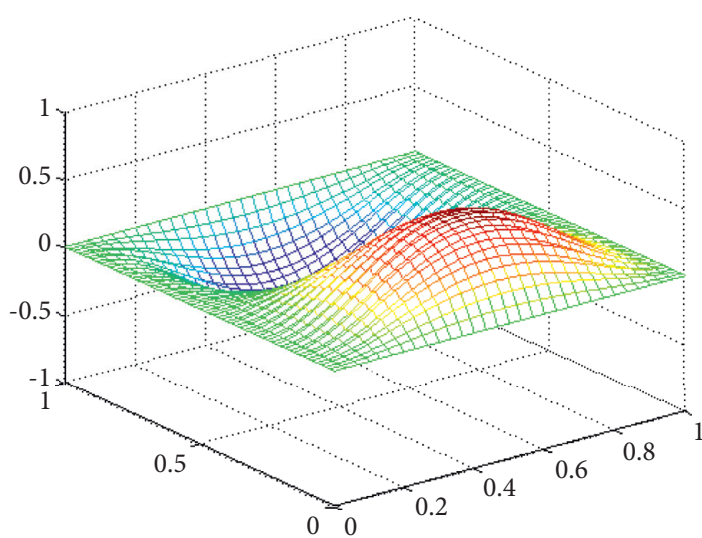

(a)

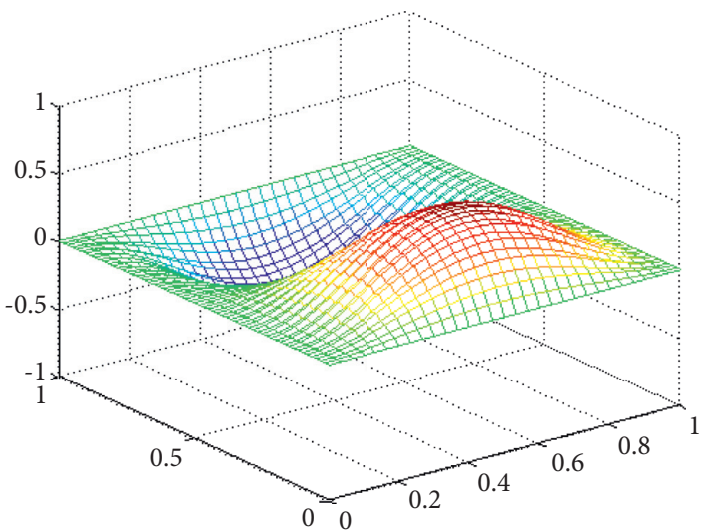

(b)

FIgURE 12: True solution and calculation solution of velocity component $u_{1}$ by the NRFE method for the Navier-Stokes equations. (a) True solution of velocity component $u_{1}$. (b) Calculation solution of velocity component $u_{1}$. 


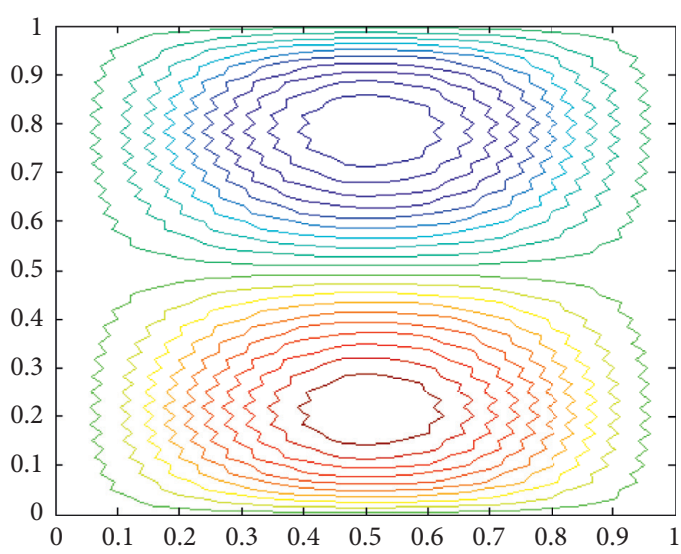

(a)

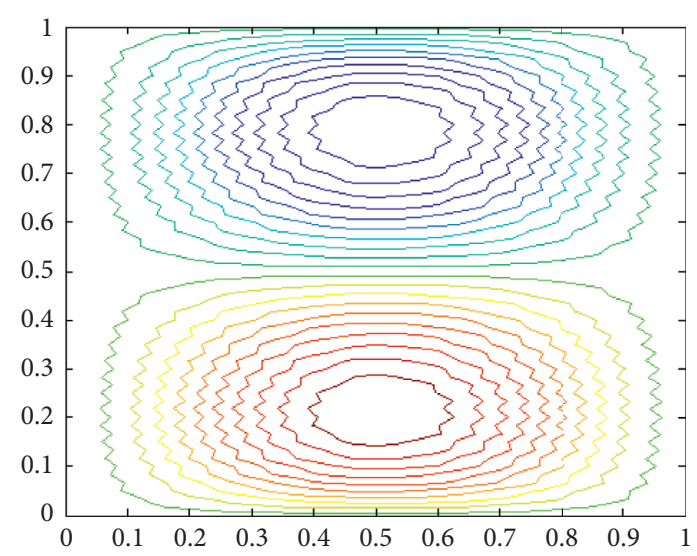

(b)

FIGURE 13: True solution contour and calculation solution contour of velocity component $u_{1}$ by the NRFE method for the Navier-Stokes equations. (a) True solution contour of velocity component $u_{1}$. (b) Calculation solution contour of velocity component $u_{1}$.

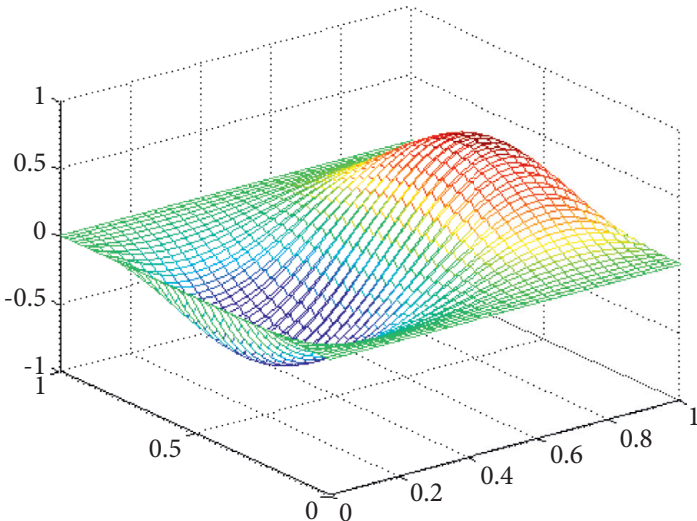

(a)

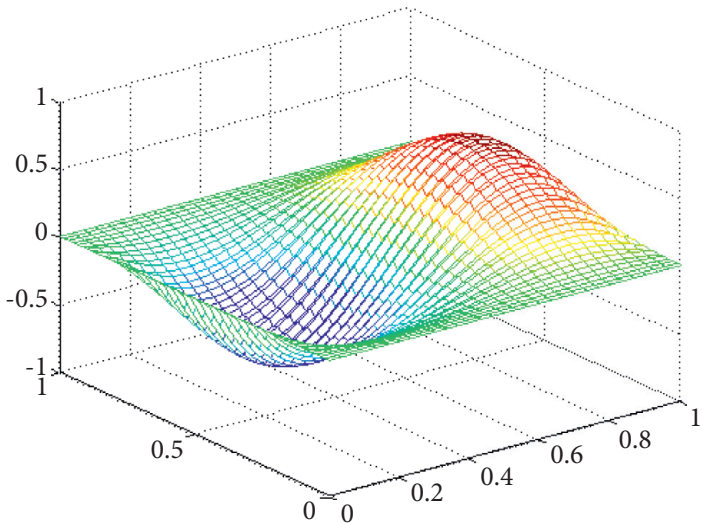

(b)

FIGURE 14: True solution and calculation solution of velocity component $u_{2}$ by the NRFE method for the Navier-Stokes equations. (a) True solution of velocity component $u_{2}$. (b) Calculation solution of velocity component $u_{2}$.

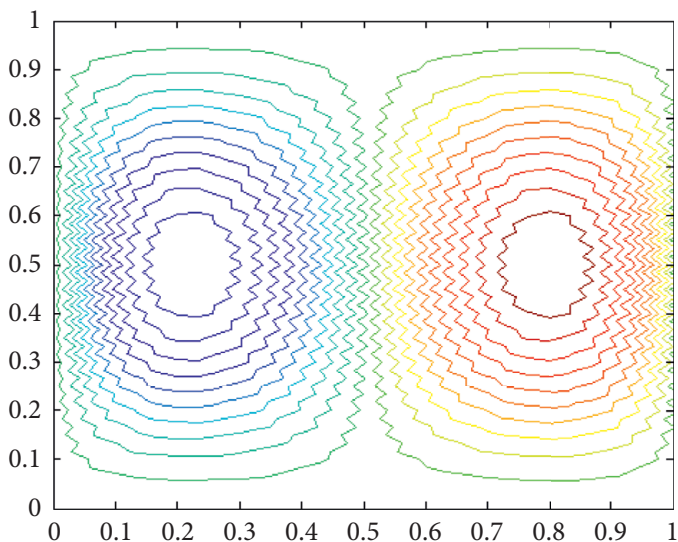

(a)

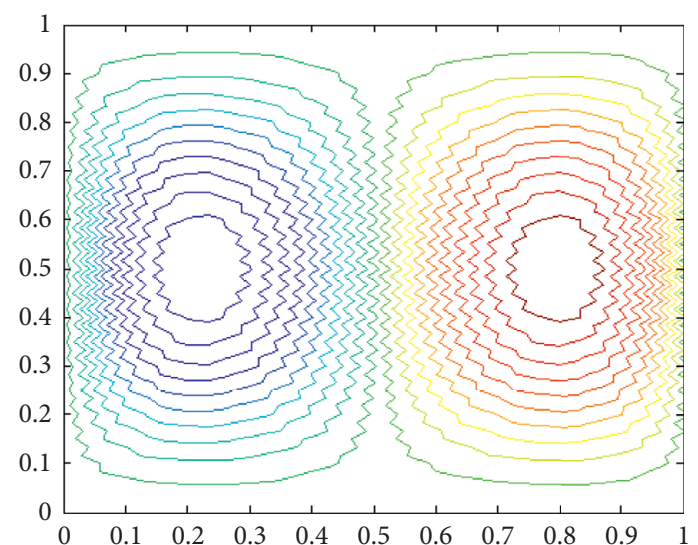

(b)

FIGURE 15: True solution contour and calculation solution contour of velocity component $u_{2}$ by the NRFE method for the Navier-Stokes equations. (a) True solution contour of velocity component $u_{2}$. (b) Calculation solution contour of velocity component $u_{2}$. 


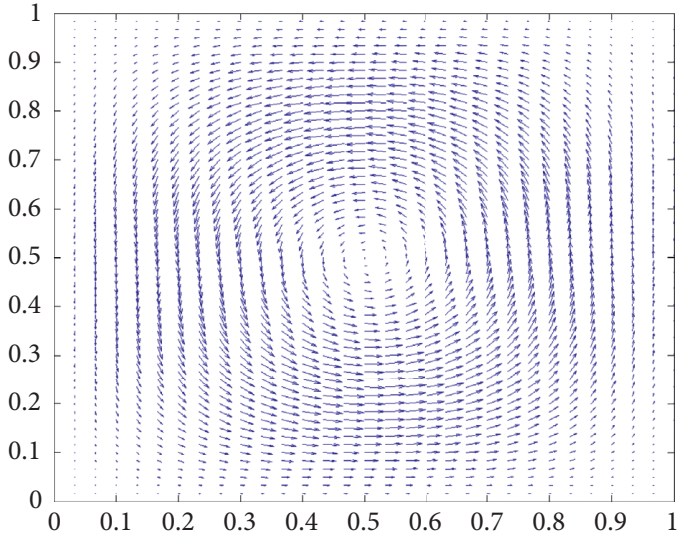

(a)

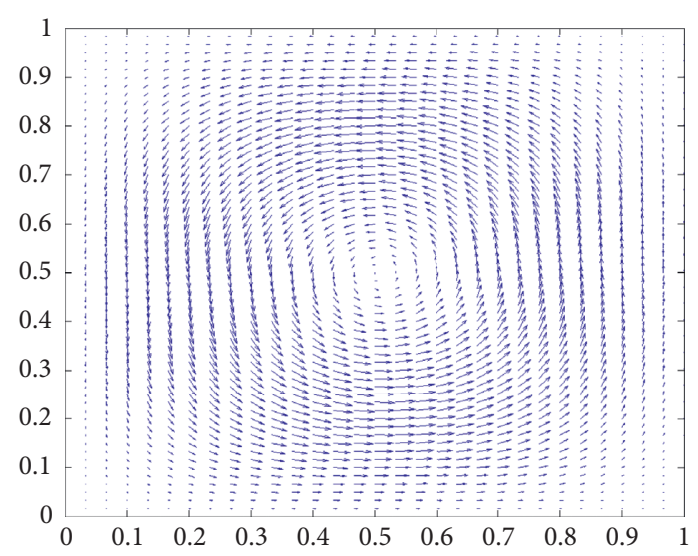

(b)

FIgURE 16: True solution vector and calculation solution vector of velocity by the NRFE method for the Navier-Stokes equations. (a) True solution vector of velocity. (b) Calculation solution vector of velocity.

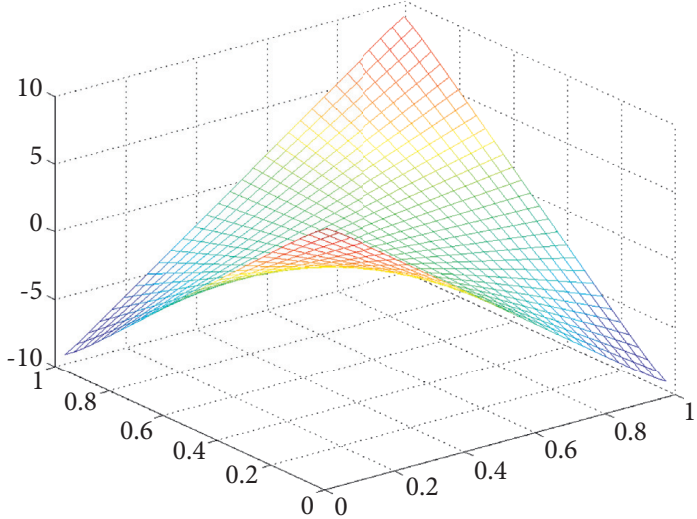

(a)

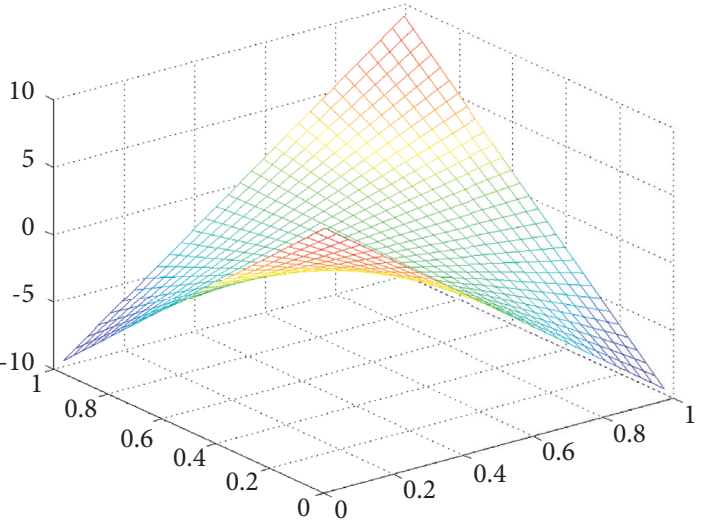

(b)

Figure 17: True solution and calculation solution of pressure by the NRFE method for the Navier-Stokes equations. (a) True solution of pressure. (b) Calculation solution of pressure.

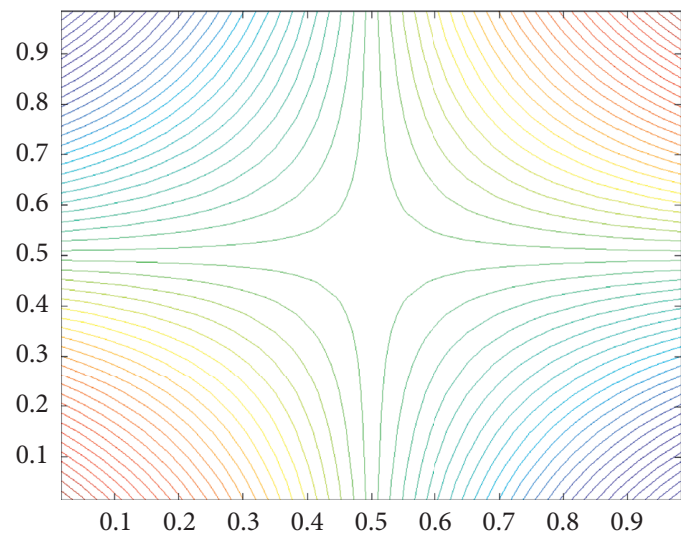

(a)

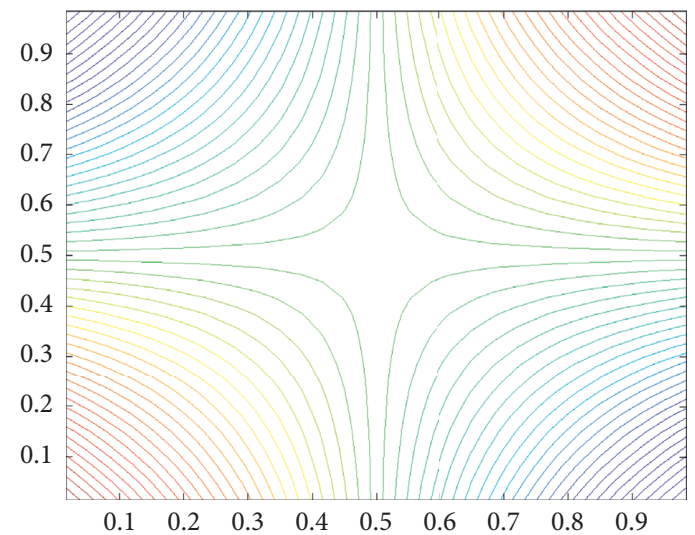

(b)

FIgURE 18: True solution contour and calculation solution contour of pressure by the NRFE method for the Navier-Stokes equations. (a) True solution contour of pressure. (b) Calculation solution contour of pressure. 
TABLE 2: Convergence study of the NRFE method for the Navier-Stokes equations in Example 2.

\begin{tabular}{lcccc}
\hline$h$ & $\mathrm{CPU}(\mathrm{s})$ & $\left\|u-u_{h}\right\|_{1, h} /\|u\|_{1, h}$ & $\left\|u-u_{h}\right\|_{0} /\|u\|_{0}$ & $\left\|p-p_{h}\right\|_{0} /\|p\|_{0}$ \\
\hline $1 / 9$ & 1 & 0.05285264668 & 0.00512070990 & 0.00660637377 \\
$1 / 16$ & 29 & 0.05062843052 & 0.00469204715 & 0.00630851030 \\
$1 / 25$ & 391 & 0.04858196977 & 0.00431486884 & 0.00603642962 \\
\hline
\end{tabular}

TABLE 3: Convergence study of the TNRFE method for the Navier-Stokes equations in Example 2.

\begin{tabular}{cccccc}
\hline$H$ & $h$ & CPU $(\mathrm{s})$ & $\left\|u-u^{h}\right\|_{1, h} /\|u\|_{1, h}$ & $\left\|u-u^{h}\right\|_{0} /\|u\|_{0}$ & $\left\|p-p^{h}\right\|_{0} /\|p\|_{0}$ \\
\hline $1 / 3$ & $1 / 9$ & 1 & 0.04494406371 & 0.00682618493 & 0.00769732820 \\
$1 / 4$ & $1 / 16$ & 16 & 0.04332036000 & 0.00617399737 & 0.00729562730 \\
$1 / 5$ & $1 / 25$ & 196 & 0.04180897072 & 0.00561062878 & 0.00693386430 \\
\hline
\end{tabular}

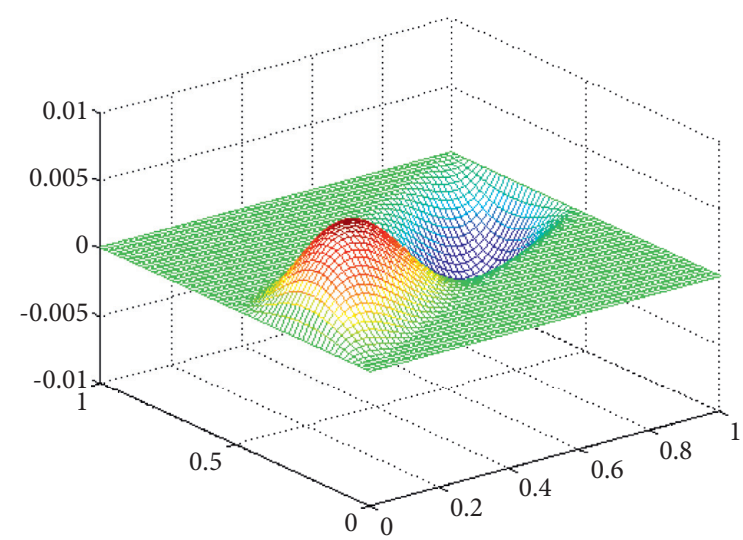

(a)

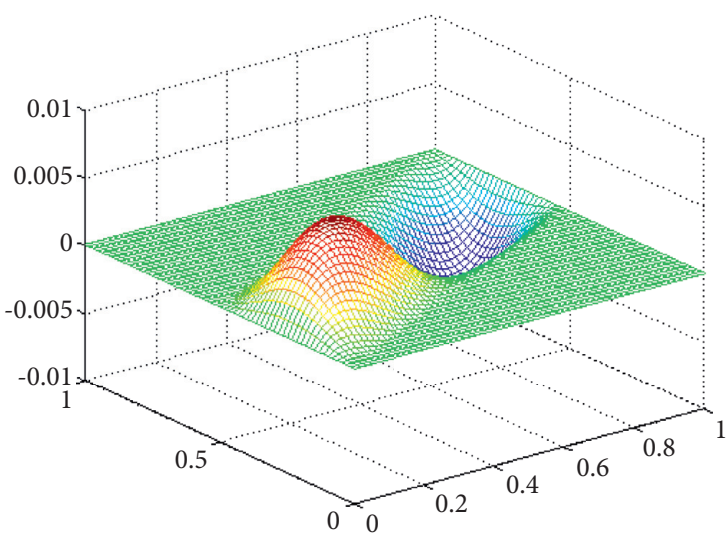

(b)

FIGURE 19: True solution and calculation solution of velocity component $u_{1}$ by the TNRFE method for the Navier-Stokes equations. (a) True solution of velocity component $u_{1}$. (b) Calculation solution of velocity component $u_{1}$.

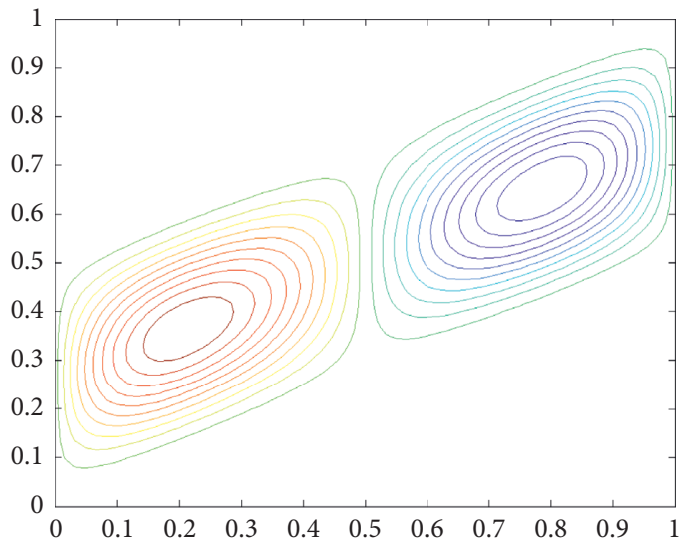

(a)

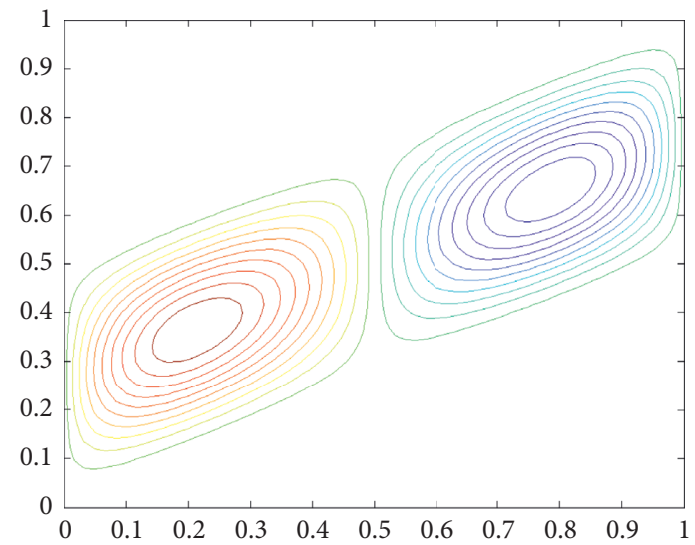

(b)

FIgURE 20: True solution contour and calculation solution contour of velocity component $u_{1}$ by the TNRFE method for the Navier-Stokes equations. (a) True solution contour of velocity component $u_{1}$. (b) Calculation solution contour of velocity component $u_{1}$. 


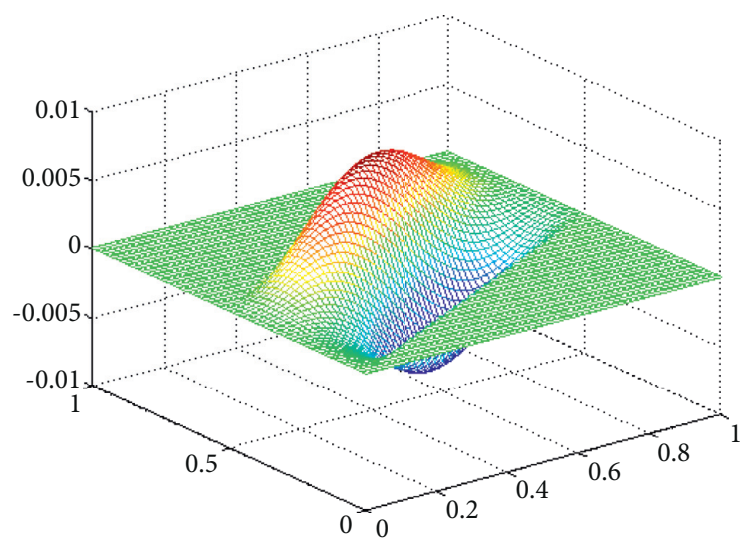

(a)

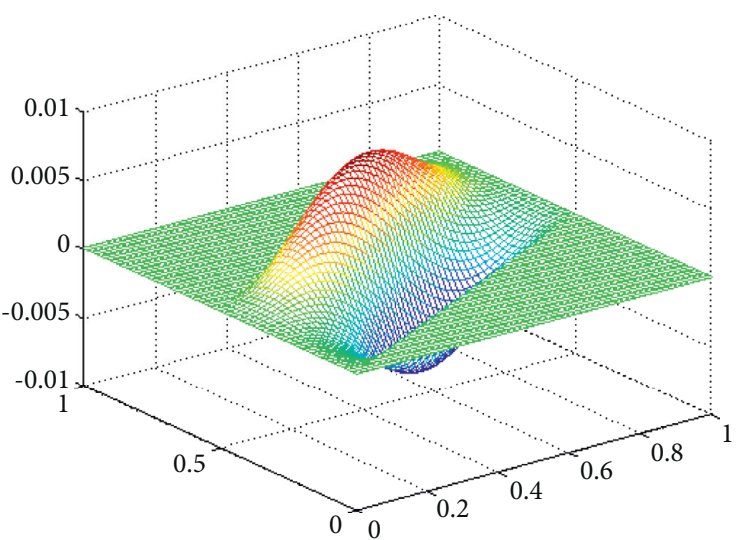

(b)

FIGURE 21: True solution and calculation solution of velocity component $u_{2}$ by the TNRFE method for the Navier-Stokes equations. (a) True solution of velocity component $u_{2}$. (b) Calculation solution of velocity component $u_{2}$.

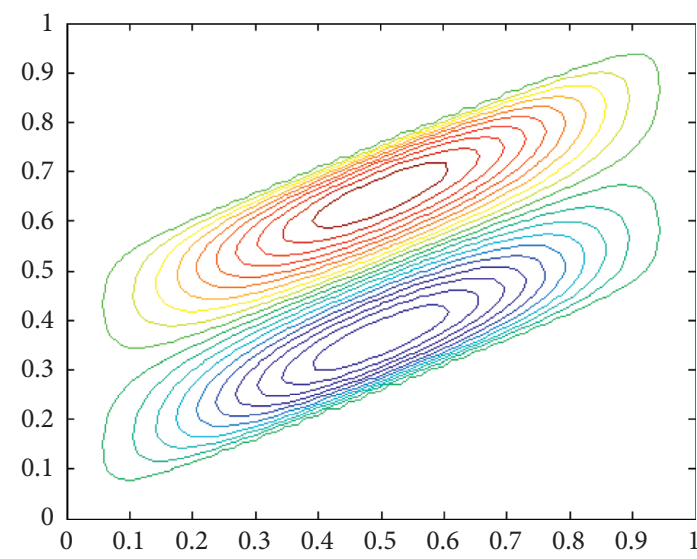

(a)

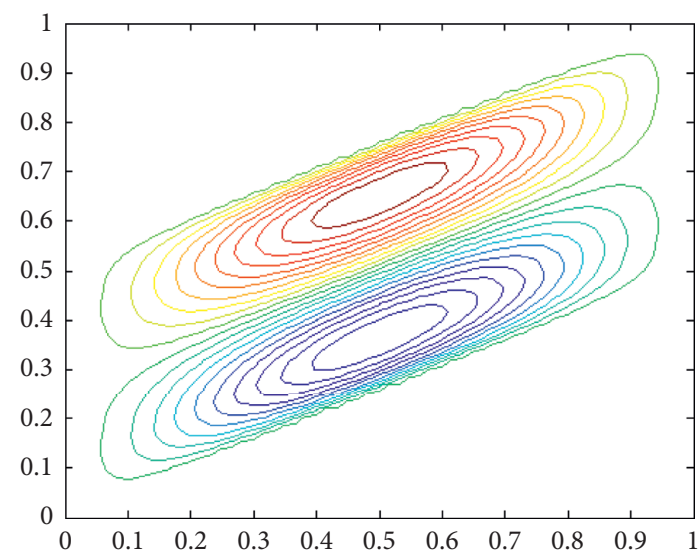

(b)

FIGURE 22: True solution contour and calculation solution contour of velocity component $u_{2}$ by the TNRFE method for the Navier-Stokes equations. (a) True solution contour of velocity component $u_{2}$. (b) Calculation solution contour of velocity component $u_{2}$.

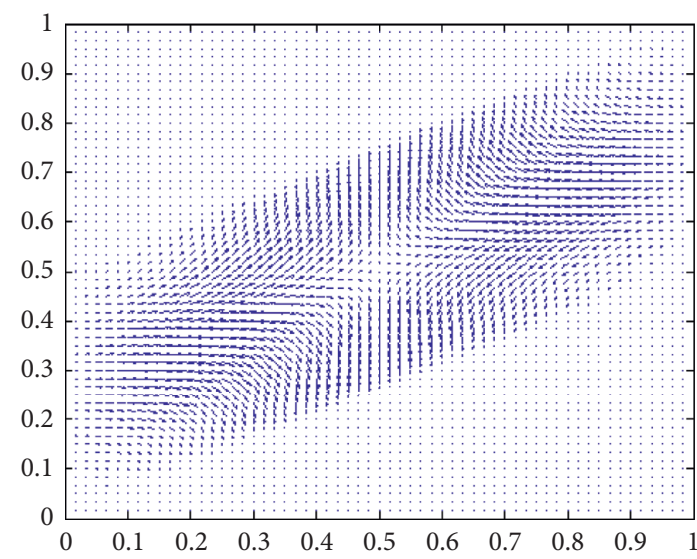

(a)

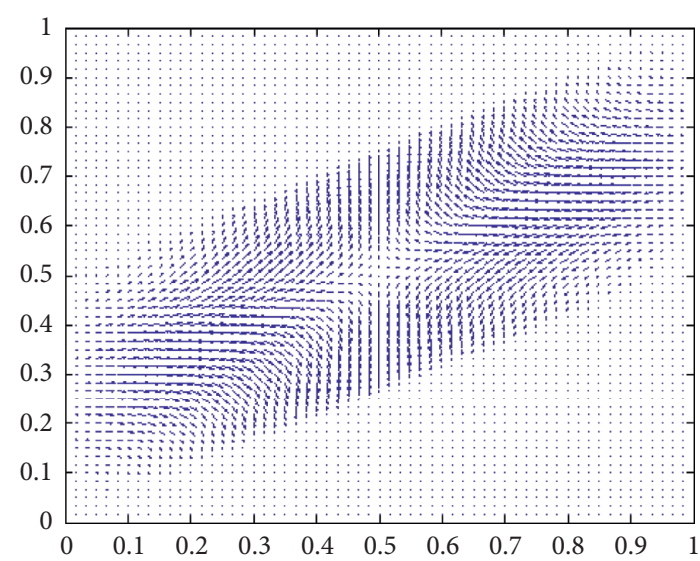

(b)

FIGURE 23: True solution vector and calculation solution vector of velocity by the TNRFE method for the Navier-Stokes equations. (a) True solution vector of velocity. (b) Calculation solution vector of velocity. 


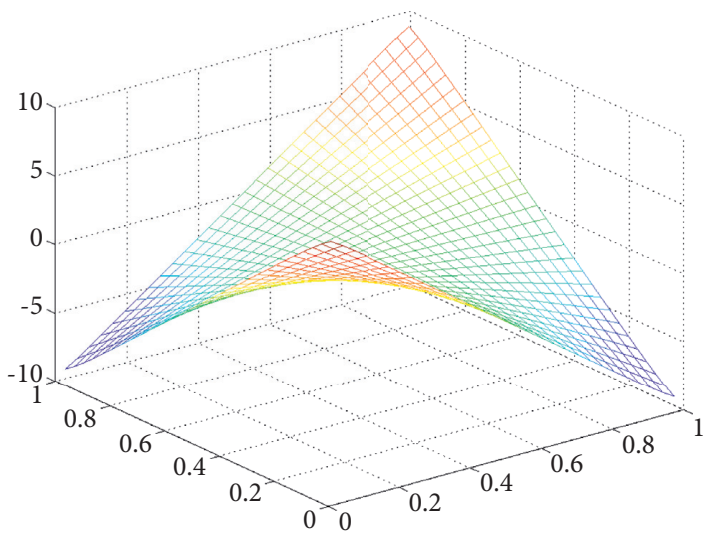

(a)

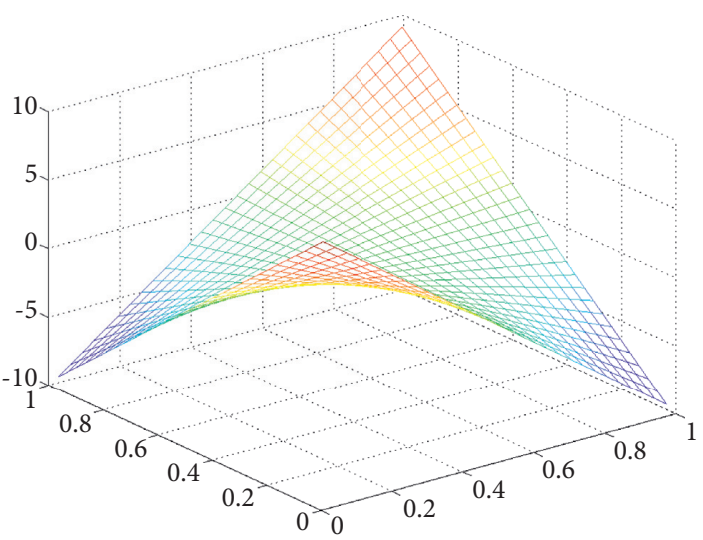

(b)

FIgURE 24: True solution and calculation solution of pressure by the TNRFE method for the Navier-Stokes equations. (a) True solution of pressure. (b) Calculation solution of pressure.

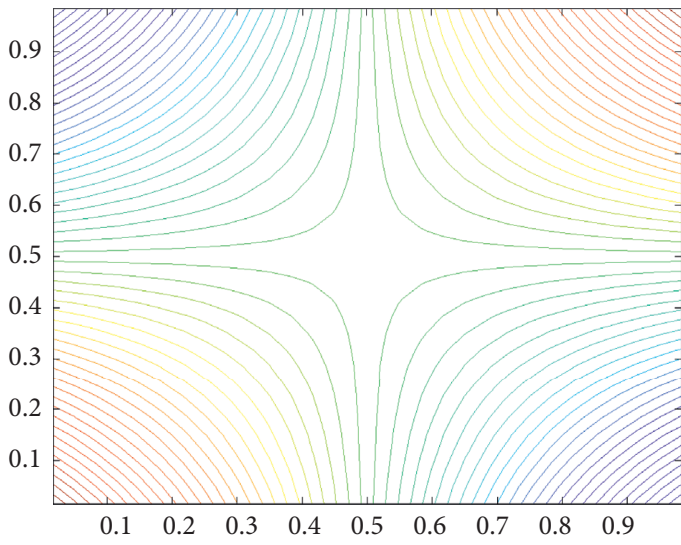

(a)

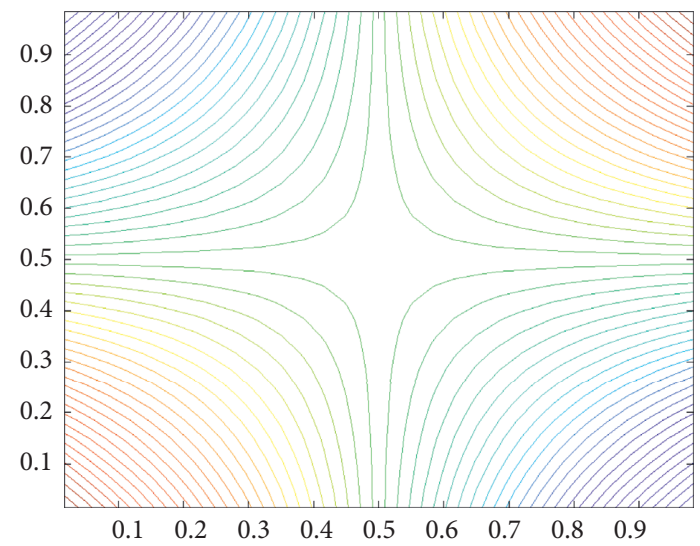

(b)

FIGURE 25: True solution contour and calculation solution contour of pressure by the TNRFE method for the Navier-Stokes equations. (a) True solution contour of pressure. (b) Calculation solution contour of pressure.

The contours of the true solution and the calculated solution of the pressure are shown in Figure 25.

\section{Conclusion}

The main work of this paper is as follows:

(1) The inequalities on the elements used to prove the standard polynomial approximation results are given.

(2) The existence, uniqueness, and convergence rate of the NRFE method for the steady Stokes equations and the Navier-Stokes equations are presented. The program design and numerical experiment results are given to verify the theory that the method has the same order of convergence speed as the conforming finite element (CFE) method.

(3) The stability and convergence rate of the Newton TNRFE method for the steady Navier-Stokes equations are proved, and the numerical results of the method are given.
Further work is as follows:

(1) The NRFE method and the TNRFE method are extended to the unsteady Navier-Stokes equations, and corresponding numerical results are obtained.

(2) The rapid development of engineering and materials science in recent years has put forward new and higher requirements for the reliability of numerical models (i.e., the existence and uniqueness of solutions, convergence, and adaptability to the change of computational background) and nonlinear behavior. The problem of overall optimization of the numerical performance of the element is presented.

\section{Data Availability}

The data used to support the findings of this study are included within the article.

\section{Conflicts of Interest}

The authors declare that they have no conflicts of interest. 


\section{Acknowledgments}

This project was supported by the Natural Science Foundation of China (no. 12171385).

\section{References}

[1] J. Jim Douglas, D. Sheen, and X. Ye, "Nonconforming Galerkin methods based on quadrilateral elements for second order elliptic problems," ESAIM: Mathematical Modelling and Numerical Analysis, vol. 33, no. 4, pp. 747-770, 1999.

[2] F. Brezzi and A. Russo, "Choosing bubbles for advectiondiffusion problems," Mathematical Models and Methods in Applied Sciences, vol. 4, no. 4, pp. 571-587, 1994.

[3] M. Crouzeix and P.-A. Raviart, "Conforming and nonconforming finite element methods for solving the stationary Stokes equations I," Revue française d'automatique informatique recherche opérationnelle. Mathématique, vol. 7, no. R3, pp. 33-75, 1973.

[4] R. Temam, Navier-Stokes Equations, The Free Encyclopedia, Amsterdam, North-Holland, 1984.

[5] R. Rannacher and S. Turek, "Simple nonconforming quadrilateral Stokes element," Numerical Methods for Partial Differential Equations, vol. 8, no. 2, pp. 97-111, 1992.

[6] H. Han, "Nonconforming elements in the mixed finite element method," Mathematics of Computation, vol. 2, pp. 223-233, 1984.

[7] Z. Cai, J. Douglas, and X. Ye, "A stable nonconforming quadrilateral finite element method for the stationary Stokes and Navier-Stokes equations," Calcolo, vol. 36, no. 4, pp. 215-232, 1999.

[8] Z. Cai, J. Douglas, J. E. Santos, D. Sheen, and X. Ye, "Nonconforming quadrilateral finite elements: ga correction," Calcolo, vol. 37, no. 4, pp. 253-254, 2000.

[9] W. Layton, "A two-level discretization method for the NavierStokes equations," Computers \& Mathematics with Applications, vol. 26, no. 2, pp. 33-38, 1993.

[10] K. Li and Y. Hou, "An aim and one-step Newton method for the Navier-Stokes equations," Computer Methods in Applied Mechanics and Engineering, vol. 190, no. 46-47, pp. 6141-6155, 2001.

[11] Y. He and K. Li, "Two-level stabilized finite element methods for the steady Navier-Stokes problem," Computing, vol. 74, no. 4, pp. 337-351, 2005.

[12] Y. He, A. Wang, Z. Chen, and K. Li, “An optimum nonlinear Galerkin method with mixed finite element for the steady Navier-Stokes equations," numer. Methods for PDEs, vol. 19, no. 2, pp. 762-775, 2003.

[13] L. Mei, "A stabilized fourier spectral method for the fractional Cahn-Hilliard equation," Computer Simulation in Application, vol. 1, no. 3, pp. 1-9, 2018.

[14] D. Shi and J. Wang, "Unconditional superconvergence analysis for nonlinear hyperbolic equation with nonconforming finite element," Applied Mathematics and Computation, vol. 305, pp. 1-16, 2017.

[15] Z. Chen, "Characteristic-nonconforming finite-element methods for advection-dominated diffusion problems," Computers \& Mathematics with Applications, vol. 48, no. 7-8, pp. 1087-1100, 2004.

[16] V. Girault and P. A. Raviart, Finite Element Methods for the Navier-Stokes Equations: Theory and Algorithms, SpringerVerlag, Berlin, Germany, 1986.

[17] O. A. Karakashian and W. N. Jureidini, "A nonconforming finite element method for the stationary Navier--Stokes equations," SIAM Journal on Numerical Analysis, vol. 35, no. 1, pp. 93-120, 1998.

[18] G. A. Baker, W. N. Jureidini, and O. A. Karakashian, "Piecewise solenoidal vector fields and the Stokes problem," SIAM Journal on Numerical Analysis, vol. 27, no. 6, pp. 1466-1485, 1990. 